\title{
Ion-Pair-Directed Borylation of Aromatic Phosphonium Salts
}

\author{
Bernadette Lee, Madalina T. Mihai, Violeta Stojalnikova, and Robert J. Phipps*(0) \\ Department of Chemistry, University of Cambridge, Lensfield Road, Cambridge CB2 1EW, United Kingdom
}

\section{Supporting Information}

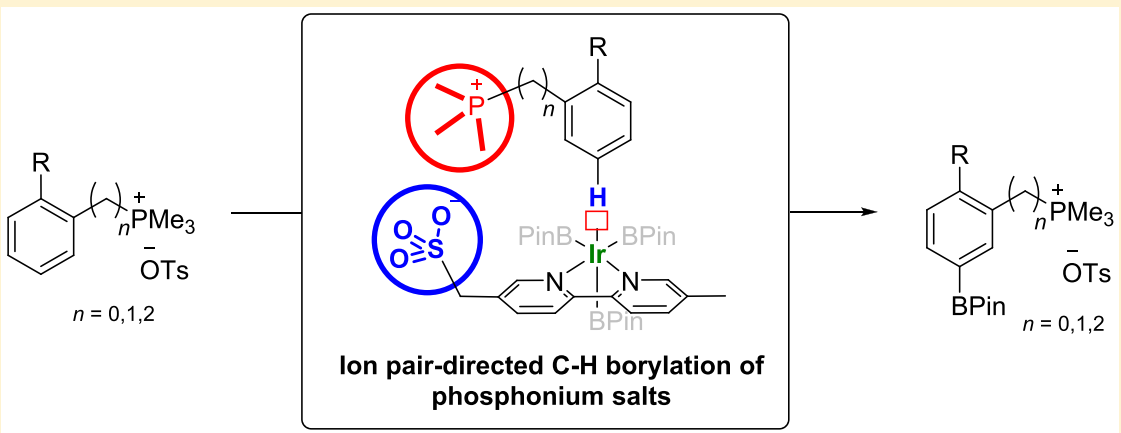

ABSTRACT: Control of positional selectivity in $\mathrm{C}-\mathrm{H}$ activation reactions remains a challenge for synthetic chemists. Noncovalent catalysis has the potential to be a powerful strategy to address this challenge. As a part of our ongoing investigations into the use of ion-pairing interactions in site-selective catalysis, we demonstrate that several classes of aromatic phosphonium salts undergo iridium-catalyzed $\mathrm{C}-\mathrm{H}$ borylation with a high selectivity for the arene meta position. This is achieved using a bifunctional bipyridine ligand bearing a pendant sulfonate group, which had previously been successful for borylation of aromatic ammonium salts. In this case, the phosphonium salts give a higher meta selectivity than the corresponding ammonium salts. We propose that the high selectivity occurs due to an attractive electrostatic interaction between the substrate and the ligand in the transition state for borylation.

$\mathrm{T}$ he direct functionalization of arene $\mathrm{C}-\mathrm{H}$ bonds using transition metal catalysis constitutes a highly effective method for elaboration of aromatic compounds. Numerous advances have been made, particularly over the last two decades. It is notable however that the majority of these advances result in a selective reaction at the arene ortho position, as a consequence of proximity to a directing group. Strategies that are able to reach further to the more remote meta and para positions are less common, and as a consequence, these positions are typically more difficult to access. $^{1}$

We and others have recently been exploring strategies that exploit a temporary noncovalent interaction between a substrate and ligand to guide the reactive transition metal to a particular position in the selectivity-determining transition state for $\mathrm{C}-\mathrm{H}$ bond functionalization. ${ }^{2}$ This approach builds on previous advances for controlling regioselectivity in reactions including aliphatic $\mathrm{C}-\mathrm{H}$ activation, ${ }^{3}$ hydroformylation, ${ }^{4}$ and others. We have been particularly interested in applying this idea to control regioselectivity in arene $\mathrm{C}-\mathrm{H}$ functionalization via $\mathrm{C}-\mathrm{H}$ borylation, which has been investigated by a number of groups. ${ }^{5-7}$ Specifically, we were curious to explore a scenario in which the catalyst engages in ion-pairing interactions with the substrate, as this is far rarer than using hydrogen bonding and relatively unexplored. ${ }^{8}$ In our previous work, we developed an anionic bipyridine ligand (1) for application in iridium-catalyzed $\mathrm{C}-\mathrm{H}$ borylation. 9 This ligand bears a pendant sulfonate group, which we hypothesized may engage in attractive electrostatic interactions with a quaternary ammonium moiety in the substrate, directing $\mathrm{C}-\mathrm{H}$ borylation to occur at the arene meta position. Gratifyingly, a high meta selectivity was obtained with a variety of chain lengths between the quaternary ammonium group and the arene, despite initial concerns that substantial substrate flexibility may be incompatible with the relatively low directionality of ion-pairing interactions. However, in these studies, we only examined quaternary ammonium salts as cationic groups on the substrates.

Phosphonium salts have a number of important chemical applications as phase transfer catalysts, ionic liquids, and lipophilic cations. They can be transformed into reactive intermediates upon deprotonation to form ylides, as widely used in the Wittig reaction and variants. ${ }^{10,11}$ Several recent studies have shown that certain phosphonium salts can also be used in cross-coupling reactions. ${ }^{12}$ Hence, we were keen to explore whether our ion-pair-directed method for controlling regioselectivity in $\mathrm{C}-\mathrm{H}$ borylation would be compatible with arenes bearing a phosphonium group, in order to demonstrate greater generality of the approach.

Special Issue: C-H Bond Functionalization

Received: March 29, 2019

Published: May 22, 2019 
We began our studies with trifluoromethyl-substituted benzyl trimethyl phosphonium salt $3 \mathbf{a}$, possessing a tosylate counterion (Table 1). An initial evaluation with the standard

Table 1. Evaluation of Ligand 1 on Benzylphosphonium Salt 3a
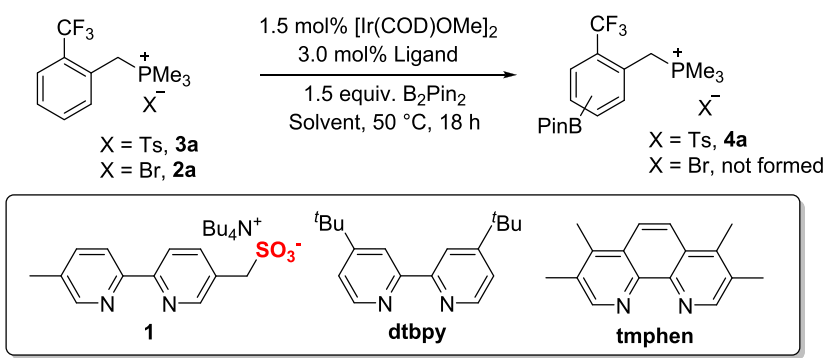

\begin{tabular}{clllcr} 
entry & $\mathrm{X}$ & ligand & \multicolumn{1}{c}{ solvent } & meta/para & $\%$ conv $^{b}$ \\
1 & OTs & dtbpy & THF & & 0 \\
2 & OTs & tmphen & THF & $1: 1.3$ & 91 \\
3 & OTs & 1 & THF & $7: 1$ & 93 \\
4 & Br & 1 & THF & & 0 \\
5 & OTs & 1 & dioxane & $>20: 1$ & 100 \\
6 & OTs & 1 & $\mathrm{CH}_{2} \mathrm{Cl}$ & $>20: 1$ & 77 \\
7 & OTs & 1 & $\mathrm{CH}_{3} \mathrm{CN}$ & $12: 1$ & 92 \\
8 & OTs & 1 & MTBE & $7: 1$ & 32 \\
9 & OTs & 1 & cyclohexane & & 0 \\
10 & OTs & tmphen & dioxane & $1: 1.6$ & 100
\end{tabular}

${ }^{a}$ Meta/para ratios are taken from the analysis of crude ${ }^{1} \mathrm{H}$ NMR spectra. ${ }^{b}$ Determined by ${ }^{1} \mathrm{H}$ NMR referenced to 1,2-dimethoxyethane as the internal standard.

borylation ligand dtbpy gave no conversion in THF at $50{ }^{\circ} \mathrm{C}$ (entry 1), but we found that switching to a more reactive tmphen ligand gave high conversion to a mixture of meta and para isomers with a poor selectivity, as expected (entry 2). We were happy to see that using our sulfonate ligand $\mathbf{1}$ in place of tmphen gave a good conversion with a 7:1 meta/para selectivity, in line with our hypothesis (entry 3 ). Under the same conditions, the same phosphonium cation but bearing bromide as the counteranion (2a) gave no conversion, presumably due to the very poor solubility of the starting material (entry 4); hence, we continued optimization using $3 a$. An evaluation of solvents revealed that in 1,4-dioxane the meta selectivity was greatly improved $(>20: 1)$ and with full conversion (entry 5 ). The selectivity was reasonably tolerant to solvent variations (entries 6-8), although nonpolar solvents were not suitable, likely due to solubility issues (entry 9). A control borylation of $\mathbf{3 a}$ in dioxane with tmphen revealed a slight bias toward para selectivity, highlighting the dramatic effect that our anionic ligand $\mathbf{1}$ has on this substrate's intrinsic selectivity toward $\mathrm{C}-\mathrm{H}$ borylation (entry 10).

With optimal conditions in hand, we proceeded to evaluate the scope of the transformation. The substrates could be synthesized very readily from substituted benzyl bromides by benzylation of trimethylphosphine, followed by anion exchange with silver tosylate, both steps proceeding with generally high yields (Scheme 1). While the use of silver is not ideal from a cost standpoint, it is also possible to access these tosylate salts from benyl tosylates (see Scheme 3).

We first examined the 2-chloro-substituted salt and found that this also gave a high meta selectivity using ligand 1 (Scheme 2, 4b). Similarly to the $\mathrm{CF}_{3}$-substituted substrate, the

Scheme 1. Synthesis of Benzyl Phosphonium Salts 2 and 3

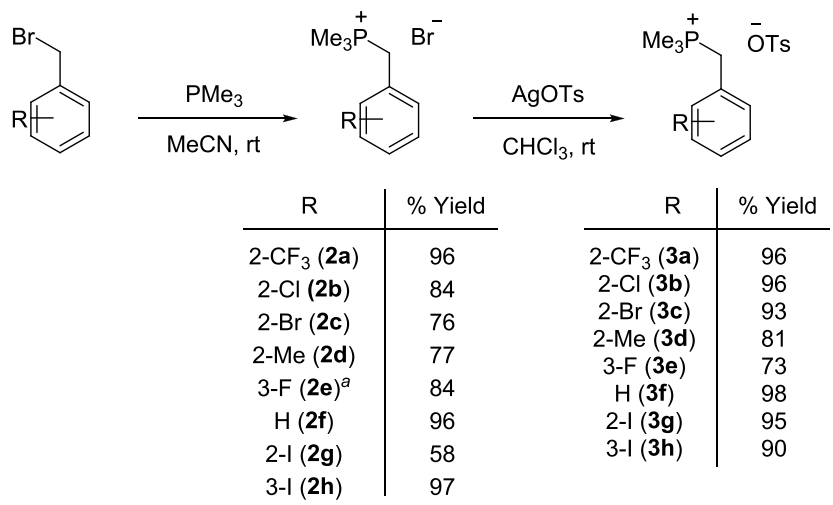

${ }^{a}$ Prepared as the chloride salt rather than the bromide salt.

Scheme 2. Scope of Substituents on Benzylphosphonium Salts 3

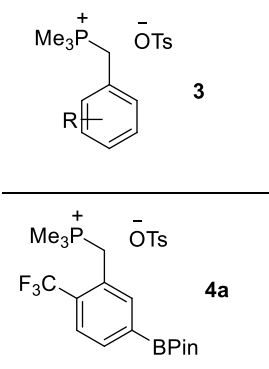

1: $89 \%$ yield $^{a},>20: 1 m: p$ (tmphen: 1:1.6 m:p)

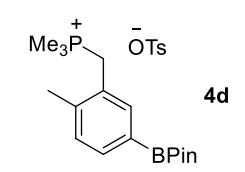

1: $89 \%$ yield $^{b}, 18: 1 m: p$ (tmphen: 1:1.3 m:p)

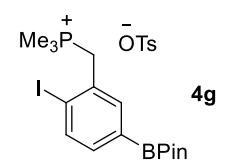

1: no reaction

(tmphen: no reaction)
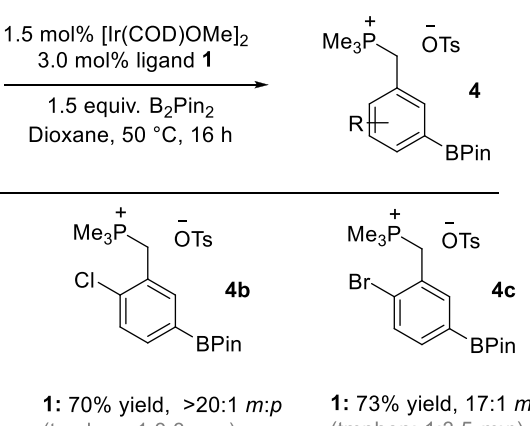
(tmphen: 1:3.3 m:p)

1: $73 \%$ yield, $17: 1 \mathrm{m:p}$ (tmphen: 1:3.5 m:p)
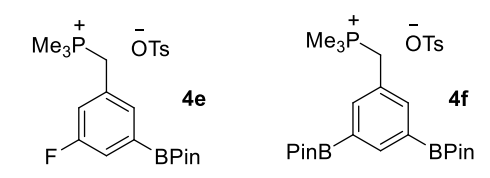
1: $68 \%$ yield, $>20: 1 m: p$ (tmphen: 1:1.3 m:p)

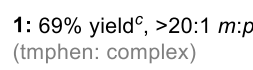

${ }^{a 1} \mathrm{H}$ NMR yield referenced to an internal standard quoted due to decomposition during purification. ${ }^{b}$ Double catalyst loadings used, reaction at $70{ }^{\circ} \mathrm{C}$. ${ }^{c}$ Three equiv of $\mathrm{B}_{2} \mathrm{Pin}_{2}$ used.

use of tmphen gave some bias toward the para selectivity, in this case, 3.3:1 para/meta (see values in parentheses). A bromo-substituted variant also worked very well, giving 17:1 $m / p$ selectivity (4c). In the case of the electron-donating methyl substituent, a higher catalyst loading of $6 \mathrm{~mol} \%$ Ir was required for good conversion, and this substrate too gave a high selectivity (4d). The small size of fluorine resulted in substrate $4 \mathbf{e}$ giving a mixture of isomers under borylation with tmphen, but with ligand $\mathbf{1}$, only the meta-borylated isomer was observed (>20:1). Finally, an unsubstituted benzylphosphonium salt also performed well (4f). In this case, it was not 
possible to stop at monoborylation, and so, 3 equiv of $\mathrm{B}_{2} \mathrm{Pin}_{2}$ was used to obtain the dimeta-borylated product in a good yield. The iodo-substituted phosphonium salts $3 \mathrm{~g}$ and $3 \mathrm{~h}$ unfortunately were found to give no conversion with either tmphen or ligand 1. Interestingly, the triarylbenzylphosphonium salt $3 \mathbf{i}$ was also found to give no reaction with either ligand. It should be mentioned that in many cases small amounts of starting material were still present at the end of the reaction, and these were impossible to separate from the borylated products as the salts were not purifiable on silica and had to be precipitated. The yields quoted have been adjusted to reflect this based on the molar mass of the starting material (see Experimental Section).

Borylation of a pyridine-derived phosphonium salt was next examined to evaluate whether selectivity between the 4- and 5positions could be obtained. In this case, the counterion exchange according to the previous substrate synthesis using silver failed in the presence of the basic pyridine. So an alternative approach was taken via the intermediate tosylate, which allows substrates to be accessed from benzyl alcohols. This approach can be advantageous for some substrates as it installs tosylate directly as the counteranion (Scheme 3a). For

Scheme 3. Synthesis and Borylation of Pyridylphosphonium Salt 5a

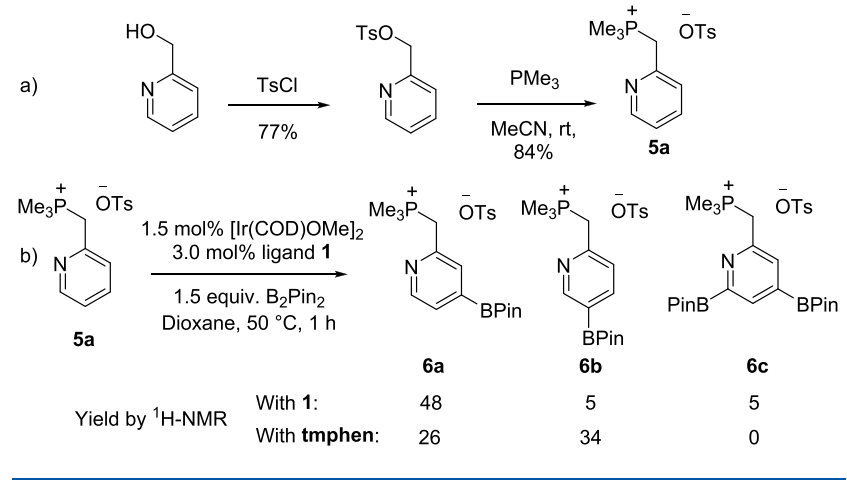

the pyridine substrate $\mathbf{5 a}$, it was quite challenging to prevent over borylation to $6 \mathrm{c}$, but by stopping the reaction after $1 \mathrm{~h}$, useful amounts of $6 \mathrm{a}$, the product of borylation at $\mathrm{C} 4$, could be obtained and the $\mathrm{C} 4 / \mathrm{C} 5$ ratio was 10:1 (corresponding to the $m / p$ ratio in nonheteroarenes). In contrast, with tmphen, the C4/C5 ratio was $\sim 1: 1$ (Scheme $3 b$ ).

We next sought to vary the carbon chain of the phosphonium salt to evaluate whether selectivity would be maintained if it is either extended or reduced. We were pleased to find that trifluoromethyl-substituted phenethyl phosphonium salt 7 a gave $>20: 1 \mathrm{~m} / \mathrm{p}$ selectivity in a good yield (Scheme 4a). In contrast, control borylation of this substrate with tmphen as a ligand gave $1: 2 \mathrm{~m} / \mathrm{p}$. While we did explore substituents apart from $\mathrm{CF}_{3}$ in this class, we found that less electron-withdrawing substituents typically gave only moderate conversions and so these were not further pursued due to the challenges of separating the product from the unreacted starting material (vide supra). Phenyltrimethyl phosphonium tosylate (7b) gave an excellent meta selectivity, resulting in dimeta-borylated product $\mathbf{8 b}$ (Scheme $4 \mathbf{b}$ ). These results provide encouragement that phosphonium salts are likely to be tolerant of a range of chain lengths, as we had previously seen with the corresponding ammonium salts, which gave a meta selectivity with both 2- and 3-carbon linker lengths. ${ }^{9}$
Scheme 4. Borylation of Longer Chain Phosphonium Salt 7a and Phenyltrimethyl Phosphonium Tosylate (7b)

(a)
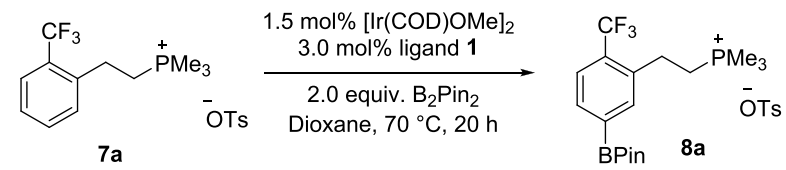

$80 \%$ yield, $>20: 1 m: p$ (tmphen: 1:2 $m: p$ )

(b)
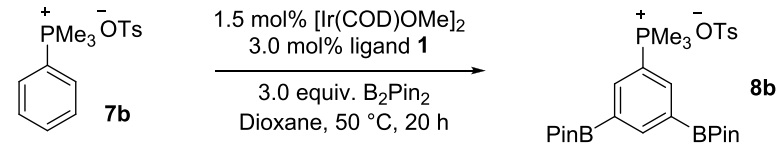

$80 \%$ yield, $>20: 1 m: p$

Finally, we demonstrate the meta-selective borylation of $3 \mathbf{b}$ on a $1.0 \mathrm{mmol}$ scale and telescope this with conversion of the BPin to a hydroxyl group followed by reduction of the phosphonium functionality with lithium aluminum hydride (Scheme 5). ${ }^{13}$ This example of further elaboration highlights the potential of our method for the rapid access to complex arene building blocks.

Scheme 5. Larger Scale Reaction and Elaboration of the Product

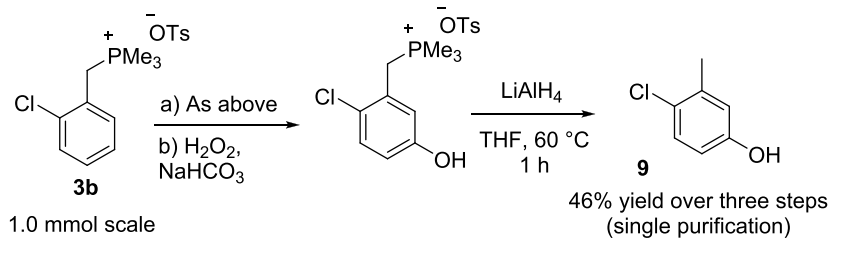

In summary, we have demonstrated that aromatic phosphonium salts are compatible with our previously reported sulfonate ligand $\mathbf{1}$ to enable $\mathrm{C}-\mathrm{H}$ borylation to be directed to the arene meta position. The selectivities are in general very high, and we envisage that this study provides further evidence of the utility of ion-pairing interactions in the design of new catalyst scaffolds for site-selective functionalization.

\section{EXPERIMENTAL SECTION}

Materials and Methods. All reagents, unless otherwise stated, were used as supplied from commercial sources without further purification. $\mathrm{CH}_{2} \mathrm{Cl}_{2}, \mathrm{THF}$, and $\mathrm{Et}_{2} \mathrm{O}$ were purified by distillation on site under an inert atmosphere via the following processes: THF and $\mathrm{Et}_{2} \mathrm{O}$ were predried over a sodium wire and then distilled from calcium hydride and lithium aluminum hydride. $\mathrm{CH}_{2} \mathrm{Cl}_{2}$ and $n$-hexane were distilled from calcium hydride.

${ }^{1} \mathrm{H}$ NMR spectra were recorded on a $600 \mathrm{MHz}$ Bruker Avance DRX-600 spectrometer, $500 \mathrm{MHz}$ Bruker DCH Cryoprobe, or 400 $\mathrm{MHz}$ QNP Cryoprobe. Chemical shifts are reported in parts per million (ppm), and the spectra are calibrated to the resonance resulting from incomplete deuteration of the solvent $\left(\mathrm{CDCl}_{3} 7.26\right.$ ppm, $\left.\mathrm{CD}_{3} \mathrm{OD} 3.31 \mathrm{ppm},\left(\mathrm{CD}_{3}\right)_{2} \mathrm{SO} 2.50 \mathrm{ppm}\right) .{ }^{13} \mathrm{C}$ NMR spectra were recorded on the same spectrometers with complete proton decoupling. Chemical shifts are reported in ppm with the solvent resonance as the internal standard $\left({ }^{13} \mathrm{CDCl}_{3} 77.16 \mathrm{ppm}, \mathrm{t} ;{ }^{13} \mathrm{CD}_{3} \mathrm{OD}\right.$ $49.00 \mathrm{ppm}$, sept; DMSO- $\left.d_{6} 39.51 \mathrm{ppm}, \mathrm{s}\right)$. Data are reported as follows: chemical shift $\delta / \mathrm{ppm}$, multiplicity $(\mathrm{s}=$ singlet, $\mathrm{d}=$ doublet, $\mathrm{t}$ = triplet, $\mathrm{q}=$ quartet, $\mathrm{br}=$ broad, $\mathrm{m}=$ multiplet or combinations thereof; ${ }^{13} \mathrm{C}$ signals are singlets unless otherwise stated), coupling 
constants $J$ in $\mathrm{Hz}$, integration ( $1 \mathrm{H}$ only). ${ }^{1} \mathrm{H}-\mathrm{COSY}, \mathrm{HSQC}, \mathrm{HMBC}$, and NOESY were used where appropriate to facilitate structural determination. The carbon attached to boron was generally not observed by ${ }^{13} \mathrm{C}$ spectroscopy due to quadrupolar relaxation. ${ }^{1} \mathrm{H}$ NMR signals are reported to 2 decimal places and ${ }^{13} \mathrm{C}$ signals to 1 decimal place ( 2 decimals places if the peaks are not distinguishable with only 1 decimal place). ${ }^{19} \mathrm{~F}$ NMR spectra were recorded on a 400 $\mathrm{MHz}$ Bruker Avance III HD spectrometer, and ${ }^{19} \mathrm{~F}$ signals are reported to 2 decimal places. ${ }^{31} \mathrm{P}$ NMR spectra were recorded on a $600 \mathrm{MHz}$ Bruker Avance DRX-600 spectrometer or a $400 \mathrm{MHz}$ Bruker Avance III HD spectrometer, and ${ }^{31} \mathrm{P}$ signals are reported to 2 decimal places.

High-resolution mass spectra (HRMS) were recorded on a Waters Micromass LCT Premier TOF spectrometer using a positive electrospray ionization (ESI+). Measured values are reported to 4 decimal places and are within $\pm 5 \mathrm{ppm}$ of the calculated value. The calculated values are based on the most abundant isotope.

Infrared (IR) spectroscopy was performed using a PerkinElmer Spectrum One FT infrared spectrophotometer sampling accessory, scanning from $4000-600 \mathrm{~cm}^{-1}$. IR absorption maxima $\left(\nu_{\max }\right)$ are reported in wavenumbers $\left(\mathrm{cm}^{-1}\right)$.

General Procedure for the Synthesis of Phosphonium 4Methylbenzenesulfonates (GP1). The corresponding phosphonium bromide (or chloride) salt and silver $p$-toluenesulfonate (1.1 equiv) were dissolved in $\mathrm{CHCl}_{3}$. The reaction mixture was stirred at room temperature for $30 \mathrm{~min}$ and then filtered through $\mathrm{MgSO}_{4}$. The filtrate was collected, and the solvent was removed under reduced pressure to afford the product.

Trimethyl(2-(trifluoromethyl)benzyl)phosphonium Bromide (2a). To a solution of 2-(trifluoromethyl)benzyl bromide (1.25 g, $5.2 \mathrm{mmol})$ in acetonitrile $(10 \mathrm{~mL})$ was added a $1.0 \mathrm{M}$ solution of trimethylphosphine in toluene $(5.8 \mathrm{~mL}, 5.8 \mathrm{mmol}, 1.1$ equiv). The reaction mixture was stirred at room temperature for $1 \mathrm{~h}$ under an argon atmosphere. The solvent was then removed under reduced pressure, and the salt precipitated with $\mathrm{CHCl}_{3}$ and $\mathrm{Et}_{2} \mathrm{O}$ (approximately a 1:10 ratio of $\mathrm{CHCl}_{3} / \mathrm{Et}_{2} \mathrm{O}$ ). The salt was collected by filtration, washed with $\mathrm{Et}_{2} \mathrm{O}$, and dried in vacuo to yield the title compound as a white powder $(1.58 \mathrm{~g}, 5.0 \mathrm{mmol}, 96 \%):{ }^{1} \mathrm{H}$ NMR $\left(600 \mathrm{MHz}, \mathrm{CDCl}_{3}\right) \delta 7.85(\mathrm{dd}, J=7.7,2.2 \mathrm{~Hz}, 1 \mathrm{H}), 7.72(\mathrm{~d}, J=7.9$ $\mathrm{Hz}, 1 \mathrm{H}), 7.64(\mathrm{t}, J=7.6 \mathrm{~Hz}, 1 \mathrm{H}), 7.49(\mathrm{t}, J=7.7 \mathrm{~Hz}, 1 \mathrm{H}), 4.37(\mathrm{~d}, J=$ $16.7 \mathrm{~Hz}, 2 \mathrm{H}), 2.24(\mathrm{~d}, J=14.2 \mathrm{~Hz}, 9 \mathrm{H}) ;{ }^{13} \mathrm{C}\left\{{ }^{1} \mathrm{H}\right\} \mathrm{NMR}(151 \mathrm{MHz}$, $\left.\mathrm{CDCl}_{3}\right) \delta 133.0\left(\mathrm{~d},{ }^{3} J_{\mathrm{C}-\mathrm{P}}=4.7 \mathrm{~Hz}\right), 128.9\left(\mathrm{~d},{ }^{5} J_{\mathrm{C}-\mathrm{P}}=3.8 \mathrm{~Hz}\right), 128.8$ $\left(\mathrm{qd},{ }^{2} J_{\mathrm{C}-\mathrm{F}}=29.8 \mathrm{~Hz},{ }^{3} J_{\mathrm{C}-\mathrm{P}}=5.7 \mathrm{~Hz}\right), 127.3-127.1(\mathrm{~m}), 126.9(\mathrm{dq}$, $\left.{ }^{3} J_{\mathrm{C}-\mathrm{F}}=1.6 \mathrm{~Hz},{ }^{2} J_{\mathrm{C}-\mathrm{P}}=9.0 \mathrm{~Hz}\right), 123.9\left(\mathrm{qd},{ }^{1} J_{\mathrm{C}-\mathrm{F}}=273.8 \mathrm{~Hz},{ }^{4} J_{\mathrm{C}-\mathrm{P}}=\right.$ $1.5 \mathrm{~Hz}), 27.9\left(\mathrm{~d},{ }^{1} J_{\mathrm{C}-\mathrm{P}}=51.1 \mathrm{~Hz}\right), 9.2\left(\mathrm{~d},{ }^{1} J_{\mathrm{C}-\mathrm{P}}=54.3 \mathrm{~Hz}\right) ;{ }^{19} \mathrm{~F}$ NMR $\left(376 \mathrm{MHz}, \mathrm{CDCl}_{3}\right) \delta-58.81 ;{ }^{31} \mathrm{P} \mathrm{NMR}\left(243 \mathrm{MHz}, \mathrm{CDCl}_{3}\right) \delta 28.29$. HRMS $\mathrm{m} / z$ (ESI+) $[\mathrm{M}-\mathrm{Br}]^{+}$calcd for $\left[\mathrm{C}_{11} \mathrm{H}_{15} \mathrm{~F}_{3} \mathrm{P}\right]^{+} 235.0858$, found 235.0849 .

Trimethyl(2-(trifluoromethyl)benzyl)phosphonium 4-Methylbenzenesulfonate ( $3 a)$. Following GP1, the compound was formed using trimethyl(2-(trifluoromethyl)benzyl)phosphonium bromide (0.53 g, $1.7 \mathrm{mmol})$, silver $p$-toluenesulfonate $(0.53 \mathrm{~g}, 1.9 \mathrm{mmol}, 1.1$ equiv), and chloroform $(5 \mathrm{~mL})$. The title compound was obtained as a white solid (0.66 g, $1.6 \mathrm{mmol}, 96 \%):{ }^{1} \mathrm{H}$ NMR $\left(600 \mathrm{MHz}, \mathrm{CDCl}_{3}\right) \delta 7.73$ $(\mathrm{d}, J=8.2 \mathrm{~Hz}, 2 \mathrm{H}), 7.69(\mathrm{~d}, J=7.9 \mathrm{~Hz}, 1 \mathrm{H}), 7.66(\mathrm{~d}, J=7.7 \mathrm{~Hz}, 1 \mathrm{H})$, $7.54(\mathrm{t}, J=7.5 \mathrm{~Hz}, 1 \mathrm{H}), 7.44(\mathrm{t}, J=7.4 \mathrm{~Hz}, 1 \mathrm{H}), 7.12(\mathrm{~d}, J=7.9 \mathrm{~Hz}$, $2 \mathrm{H}), 4.06(\mathrm{~d}, J=16.8 \mathrm{~Hz}, 2 \mathrm{H}), 2.31(\mathrm{~s}, 3 \mathrm{H}), 2.08(\mathrm{~d}, J=14.4 \mathrm{~Hz}$, $9 \mathrm{H}) ;{ }^{13} \mathrm{C}\left\{{ }^{1} \mathrm{H}\right\} \operatorname{NMR}\left(151 \mathrm{MHz}, \mathrm{CDCl}_{3}\right) \delta 143.6,139.4,133.2(\mathrm{~d}$, $\left.{ }^{3} J_{\mathrm{C}-\mathrm{P}}=4.8 \mathrm{~Hz}\right), 133.0\left(\mathrm{~d},{ }^{4} J_{\mathrm{C}-\mathrm{P}}=2.3 \mathrm{~Hz}\right), 128.9\left(\mathrm{qd},{ }^{2} \mathrm{~J}_{\mathrm{C}-\mathrm{F}}=30.2 \mathrm{~Hz}\right.$, $\left.{ }^{3} J_{\mathrm{C}-\mathrm{P}}=5.7 \mathrm{~Hz}\right), 128.72,128.65\left(\mathrm{~d},{ }^{5} J_{\mathrm{C}-\mathrm{P}}=3.5 \mathrm{~Hz}\right), 127.3\left(\mathrm{qd},{ }^{3} J_{\mathrm{C}-\mathrm{F}}=\right.$ $\left.1.7 \mathrm{~Hz},{ }^{2} J_{\mathrm{C}-\mathrm{P}}=8.7 \mathrm{~Hz}\right), 127.1(\mathrm{~m}), 125.8,124.0\left(\mathrm{qd},{ }^{1} \mathrm{~J}_{\mathrm{C}-\mathrm{F}}=273.3\right.$ $\left.\mathrm{Hz},{ }^{4} J_{\mathrm{C}-\mathrm{P}}=1.6 \mathrm{~Hz}\right), 27.7\left(\mathrm{~d},{ }^{1} J_{\mathrm{C}-\mathrm{P}}=50.3 \mathrm{~Hz}\right), 21.3,8.4\left(\mathrm{~d},{ }^{1} J_{\mathrm{C}-\mathrm{P}}=\right.$ $54.1 \mathrm{~Hz}) ;{ }^{19} \mathrm{~F}$ NMR $\left(376 \mathrm{MHz}, \mathrm{CDCl}_{3}\right) \delta-58.86 ;{ }^{31} \mathrm{P}$ NMR $(243$ $\left.\mathrm{MHz}_{\mathrm{CDCl}}\right) \delta$ 29.07; HRMS $\mathrm{m} / z$ (ESI+) $[\mathrm{M}-\mathrm{OTs}]^{+}$calcd for $\left[\mathrm{C}_{11} \mathrm{H}_{15} \mathrm{~F}_{3} \mathrm{P}\right]^{+}$235.0858, found 235.0853.

(2-Chlorobenzyl)trimethylphosphonium Bromide (2b). To a solution of 2-chlorobenzyl bromide $(0.65 \mathrm{~mL}, 5 \mathrm{mmol})$ in acetonitrile $(10 \mathrm{~mL})$ was added a $1.0 \mathrm{M}$ solution of trimethylphosphine in THF (5.5 mL, $5.5 \mathrm{mmol}, 1.1$ equiv) dropwise. The reaction mixture was stirred at room temperature for $1 \mathrm{~h}$ under an argon atmosphere. The solvent was then removed, and the salt precipitated with $\mathrm{Et}_{2} \mathrm{O}$. The salt was collected by filtration, washed with $\mathrm{Et}_{2} \mathrm{O}$, and dried in vacuo to yield the title compound as a white powder $(1.18 \mathrm{~g}, 4.2 \mathrm{mmol}$, 84\%): ${ }^{1} \mathrm{H}$ NMR $\left(600 \mathrm{MHz}, \mathrm{CDCl}_{3}\right) \delta 7.74-7.72(\mathrm{~m}, 1 \mathrm{H}), 7.40-7.39$ $(\mathrm{m}, 1 \mathrm{H}), 7.26-7.29(\mathrm{~m}, 2 \mathrm{H}), 4.32(\mathrm{~d}, J=16.2 \mathrm{~Hz}, 2 \mathrm{H}), 2.21(\mathrm{~d}, J=$ $14.2 \mathrm{~Hz}, 9 \mathrm{H}) ;{ }^{13} \mathrm{C}\left\{{ }^{1} \mathrm{H}\right\}$ NMR $\left(151 \mathrm{MHz}, \mathrm{CDCl}_{3}\right) \delta 133.9\left(\mathrm{~d},{ }^{3} \mathrm{~J}_{\mathrm{C}-\mathrm{P}}=\right.$ $6.2 \mathrm{~Hz}), 132.7\left(\mathrm{~d},{ }^{3} J_{\mathrm{C}-\mathrm{P}}=5.0 \mathrm{~Hz}\right), 130.3\left(\mathrm{~d},{ }^{5} J_{\mathrm{C}-\mathrm{P}}=3.3 \mathrm{~Hz}\right), 130.1$ $\left(\mathrm{d},{ }^{4} J_{\mathrm{C}-\mathrm{P}}=3.9 \mathrm{~Hz}\right), 128.0\left(\mathrm{~d},{ }^{4} J_{\mathrm{C}-\mathrm{P}}=3.5 \mathrm{~Hz}\right), 126.9\left(\mathrm{~d},{ }^{2} J_{\mathrm{C}-\mathrm{P}}=9.3\right.$ $\mathrm{Hz}), 28.1\left(\mathrm{~d},{ }^{1} J_{\mathrm{C}-\mathrm{P}}=50.1 \mathrm{~Hz}\right), 9.1\left(\mathrm{~d},{ }^{1} J_{\mathrm{C}-\mathrm{P}}=54.0 \mathrm{~Hz}\right) ;{ }^{31} \mathrm{P}$ NMR $\left(243 \mathrm{MHz}, \mathrm{CDCl}_{3}\right) \delta 28.82$. HRMS $\mathrm{m} / z$ (ESI+) $[\mathrm{M}-\mathrm{Br}]^{+}$calcd for $\left[\mathrm{C}_{10} \mathrm{H}_{15} \mathrm{ClP}\right]^{+}$201.0594, found 201.0587 .

(2-Chlorobenzyl)trimethylphosphonium 4-Methylbenzenesulfonate (3b). Following GP1, the compound was formed using (2chlorobenzyl)trimethylphosphonium bromide $(0.56 \mathrm{~g}, 2 \mathrm{mmol})$, silver $p$-toluenesulfonate $(0.61 \mathrm{~g}, 2.2 \mathrm{mmol}, 1.1$ equiv), and chloroform (5 $\mathrm{mL})$. The title compound was obtained as a white solid $(0.72 \mathrm{~g}, 1.9$ mmol, 96\%): ${ }^{1} \mathrm{H}$ NMR (600 MHz, $\left.\mathrm{CDCl}_{3}\right) \delta 7.75(\mathrm{~d}, J=8.2 \mathrm{~Hz}$, $2 \mathrm{H}), 7.54-7.52(\mathrm{~m}, 1 \mathrm{H}), 7.39(\mathrm{~d}, J=7.4 \mathrm{~Hz}, 1 \mathrm{H}), 7.28-7.22(\mathrm{~m}$, $2 \mathrm{H}), 7.14(\mathrm{~d}, J=7.9 \mathrm{~Hz}, 2 \mathrm{H}), 4.04(\mathrm{~d}, J=16.4 \mathrm{~Hz}, 2 \mathrm{H}), 2.32(\mathrm{~s}, 3 \mathrm{H})$, $2.08(\mathrm{~d}, J=14.4 \mathrm{~Hz}, 9 \mathrm{H}) ;{ }^{13} \mathrm{C}\left\{{ }^{1} \mathrm{H}\right\}$ NMR $\left(151 \mathrm{MHz}, \mathrm{CDCl}_{3}\right) \delta$ 143.6, 139.5, $133.8\left(\mathrm{~d},{ }^{3} \mathrm{~J}_{\mathrm{C}-\mathrm{P}}=6.1 \mathrm{~Hz}\right), 132.8\left(\mathrm{~d},{ }^{3} \mathrm{~J}_{\mathrm{C}-\mathrm{P}}=4.9 \mathrm{~Hz}\right)$, $130.2\left(\mathrm{~d},{ }^{5} J_{\mathrm{C}-\mathrm{P}}=3.3 \mathrm{~Hz}\right), 129.9\left(\mathrm{~d},{ }^{4} \mathrm{~J}_{\mathrm{C}-\mathrm{P}}=3.9 \mathrm{~Hz}\right), 128.8,128.0(\mathrm{~d}$, $\left.{ }^{4} J_{\mathrm{C}-\mathrm{P}}=3.5 \mathrm{~Hz}\right), 127.3\left(\mathrm{~d},{ }^{2} J_{\mathrm{C}-\mathrm{P}}=9.3 \mathrm{~Hz}\right), 125.8,27.8\left(\mathrm{~d},{ }^{1} J_{\mathrm{C}-\mathrm{P}}=49.8\right.$ $\mathrm{Hz}), 21.3,8.2\left(\mathrm{~d},{ }^{1} J_{\mathrm{C}-\mathrm{P}}=53.8 \mathrm{~Hz}\right) ;{ }^{31} \mathrm{P} \mathrm{NMR}\left(243 \mathrm{MHz}^{\mathrm{CDCl}} \mathrm{CDC}_{3}\right) \delta$ 29.51; HRMS $\mathrm{m} / z$ (ESI $)[\mathrm{M}-\mathrm{OTs}]^{+}$calcd for $\left[\mathrm{C}_{10} \mathrm{H}_{15} \mathrm{ClP}\right]^{+}$ 201.0594, found 201.0596.

(2-Bromobenzyl)trimethylphosphonium Bromide (2c). To a solution of 2-bromobenzyl bromide $(1.25 \mathrm{~g}, 5 \mathrm{mmol})$ in acetonitrile $(10 \mathrm{~mL})$ was added a $1.0 \mathrm{M}$ solution of trimethylphosphine in THF (5.5 mL, $5.5 \mathrm{mmol}, 1.1$ equiv) dropwise. The reaction mixture was stirred at room temperature for 15 min under an argon atmosphere, with the product being observed to precipitate from solution. The reaction mixture was then filtered, and the solids were washed with $\mathrm{Et}_{2} \mathrm{O}$ and then dried in vacuo to yield the title compound as a white powder $(1.24 \mathrm{~g}, 3.8 \mathrm{mmol}, 76 \%):{ }^{1} \mathrm{H}$ NMR $\left(600 \mathrm{MHz}, \mathrm{CDCl}_{3}\right) \delta$ $7.77-7.75(\mathrm{~m}, 1 \mathrm{H}), 7.61(\mathrm{~d}, J=8.0 \mathrm{~Hz}, 1 \mathrm{H}), 7.36(\mathrm{t}, J=7.5 \mathrm{~Hz}, 1 \mathrm{H})$, $7.22(\mathrm{tt}, J=7.7,2.1 \mathrm{~Hz}, 1 \mathrm{H}), 4.37(\mathrm{~d}, J=16.2 \mathrm{~Hz}, 2 \mathrm{H}), 2.25(\mathrm{~d}, J=$ $14.2 \mathrm{~Hz}, 9 \mathrm{H}) ;{ }^{13} \mathrm{C}\left\{{ }^{1} \mathrm{H}\right\} \mathrm{NMR}\left(151 \mathrm{MHz}, \mathrm{CDCl}_{3}\right) \delta 133.7\left(\mathrm{~d},{ }^{4} J_{\mathrm{C}-\mathrm{P}}=\right.$ $3.2 \mathrm{~Hz}), 132.7\left(\mathrm{~d},{ }^{3} J_{\mathrm{C}-\mathrm{P}}=4.9 \mathrm{~Hz}\right), 130.3\left(\mathrm{~d},{ }^{4} J_{\mathrm{C}-\mathrm{P}}=3.9 \mathrm{~Hz}\right), 128.7$ $\left(\mathrm{d},{ }^{2} J_{\mathrm{C}-\mathrm{P}}=9.2 \mathrm{~Hz}\right), 128.6\left(\mathrm{~d},{ }^{5} J_{\mathrm{C}-\mathrm{P}}=3.6 \mathrm{~Hz}\right), 124.5\left(\mathrm{~d},{ }^{3} J_{\mathrm{C}-\mathrm{P}}=6.5\right.$ $\mathrm{Hz}), 30.5\left(\mathrm{~d},{ }^{1} J_{\mathrm{C}-\mathrm{P}}=50.0 \mathrm{~Hz}\right), 9.1\left(\mathrm{~d},{ }^{1} J_{\mathrm{C}-\mathrm{P}}=54.0 \mathrm{~Hz}\right) ;{ }^{31} \mathrm{P}$ NMR $\left(243 \mathrm{MHz} \mathrm{CDCl}_{3}\right) \delta 28.80$. HRMS $\mathrm{m} / z$ (ESI+) $[\mathrm{M}-\mathrm{Br}]^{+}$calcd for $\left[\mathrm{C}_{10} \mathrm{H}_{15} \mathrm{BrP}\right]^{+} 245.0089$, found 245.0082 .

(2-Bromobenzyl)trimethylphosphonium 4-Methylbenzenesulfonate (3c). Following GP1, the compound was formed using (2bromobenzyl)trimethylphosphonium bromide $(0.65 \mathrm{~g}, 2 \mathrm{mmol})$, silver $p$-toluenesulfonate $(0.61 \mathrm{~g}, 2.2 \mathrm{mmol}, 1.1 \mathrm{equiv})$, and chloroform $(5 \mathrm{~mL})$. The title compound was obtained as a white solid (0.78 g, $1.9 \mathrm{mmol}, 93 \%):{ }^{1} \mathrm{H}$ NMR $\left(600 \mathrm{MHz}, \mathrm{CDCl}_{3}\right) \delta 7.76$ $(\mathrm{d}, J=8.2 \mathrm{~Hz}, 2 \mathrm{H}), 7.59-7.56(\mathrm{~m}, 1 \mathrm{H}), 7.30(\mathrm{t}, J=7.5 \mathrm{~Hz}, 2 \mathrm{H}), 7.18$ $(\mathrm{tt}, J=7.8,2.1 \mathrm{~Hz}, 1 \mathrm{H}), 7.14-7.13(\mathrm{~d}, J=7.9 \mathrm{~Hz}, 2 \mathrm{H}), 4.10(\mathrm{~d}, J=$ 16.3 Hz, 2H), $2.33(\mathrm{~s}, 3 \mathrm{H}), 2.12(\mathrm{~d}, J=14.3 \mathrm{~Hz}, 9 \mathrm{H}) ;{ }^{13} \mathrm{C}\left\{{ }^{1} \mathrm{H}\right\}$ NMR $\left(151 \mathrm{MHz}, \mathrm{CDCl}_{3}\right) \delta 143.6,139.5,133.6\left(\mathrm{~d},{ }^{4} J_{\mathrm{C}-\mathrm{P}}=3.3 \mathrm{~Hz}\right), 132.7$ $\left(\mathrm{d},{ }^{3} J_{\mathrm{C}-\mathrm{P}}=4.8 \mathrm{~Hz}\right), 130.1\left(\mathrm{~d},{ }^{4} J_{\mathrm{C}-\mathrm{P}}=3.9 \mathrm{~Hz}\right), 129.1\left(\mathrm{~d},{ }^{2} J_{\mathrm{C}-\mathrm{P}}=9.2\right.$ $\mathrm{Hz}), 128.8,128.6\left(\mathrm{~d},{ }^{5} J_{\mathrm{C}-\mathrm{P}}=3.5 \mathrm{~Hz}\right), 125.8,124.5\left(\mathrm{~d},{ }^{3} J_{\mathrm{C}-\mathrm{P}}=6.4\right.$ $\mathrm{Hz}), 30.3\left(\mathrm{~d},{ }^{1} J_{\mathrm{C}-\mathrm{P}}=49.7 \mathrm{~Hz}\right), 21.3,8.3\left(\mathrm{~d},{ }^{1} J_{\mathrm{C}-\mathrm{P}}=53.8 \mathrm{~Hz}\right) ;{ }^{31} \mathrm{P}$ NMR $\left(243 \mathrm{MHz}, \mathrm{CDCl}_{3}\right) \delta 29.32$; HRMS $m / z(\mathrm{ESI}+)[\mathrm{M}-\mathrm{OTs}]^{+}$ calcd for $\left[\mathrm{C}_{10} \mathrm{H}_{15} \mathrm{BrP}\right]^{+} 245.0089$, found 245.0086 .

Trimethyl(2-methylbenzyl)phosphonium Bromide (2d). To a solution of 2-methylbenzyl bromide $(0.67 \mathrm{~mL}, 5 \mathrm{mmol})$ in acetonitrile $(10 \mathrm{~mL})$ was added a $1.0 \mathrm{M}$ solution of trimethylphosphine in THF (5.5 mL, $5.5 \mathrm{mmol}, 1.1$ equiv) dropwise. The reaction mixture was stirred at room temperature for $1 \mathrm{~h}$ under an argon atmosphere, with the product being observed to precipitate from solution. The reaction mixture was then filtered, and the solids were washed with $\mathrm{Et}_{2} \mathrm{O}$ and then dried in vacuo to yield the title compound as a white powder (1.01 g, $3.9 \mathrm{mmol}, 77 \%):{ }^{1} \mathrm{H} \mathrm{NMR}\left(600 \mathrm{MHz}, \mathrm{CDCl}_{3}\right) \delta 7.37$ (dd, $J$ $=7.6,2.7 \mathrm{~Hz}, 1 \mathrm{H}), 7.24(\mathrm{~m}, 2 \mathrm{H}), 7.21-7.19(\mathrm{~m}, 1 \mathrm{H}), 4.20(\mathrm{~d}, J=$ $16.3 \mathrm{~Hz}, 2 \mathrm{H}), 2.43(\mathrm{~d}, J=1.4 \mathrm{~Hz}, 3 \mathrm{H}), 2.21(\mathrm{~d}, J=14.2 \mathrm{~Hz}, 9 \mathrm{H})$; ${ }^{13} \mathrm{C}\left\{{ }^{1} \mathrm{H}\right\}$ NMR $\left(151 \mathrm{MHz}, \mathrm{CDCl}_{3}\right) \delta 137.0\left(\mathrm{~d}^{3}{ }^{3} \mathrm{C}_{\mathrm{C}-\mathrm{P}}=5.7 \mathrm{~Hz}\right), 131.5$ 
$\left(\mathrm{d},{ }^{5} J_{\mathrm{C}-\mathrm{P}}=3.4 \mathrm{~Hz}\right), 131.2\left(\mathrm{~d},{ }^{3} J_{\mathrm{C}-\mathrm{P}}=5.0 \mathrm{~Hz}\right), 128.5\left(\mathrm{~d},{ }^{4} J_{\mathrm{C}-\mathrm{P}}=4.0\right.$ $\mathrm{Hz}), 126.7(\mathrm{~m}), 27.8\left(\mathrm{~d},{ }^{1} J_{\mathrm{C}-\mathrm{P}}=49.4 \mathrm{~Hz}\right), 20.7\left(\mathrm{~d},{ }^{4} J_{\mathrm{C}-\mathrm{P}}=1.3 \mathrm{~Hz}\right)$, $8.8\left(\mathrm{~d},{ }^{1} J_{\mathrm{C}-\mathrm{P}}=54.1 \mathrm{~Hz}\right) ;{ }^{31} \mathrm{P}$ NMR $\left(243 \mathrm{MHz}, \mathrm{CDCl}_{3}\right) \delta 28.25$. HRMS $m / z$ (ESI +$)[\mathrm{M}-\mathrm{Br}]^{+}$calcd for $\left[\mathrm{C}_{11} \mathrm{H}_{18} \mathrm{P}\right]^{+} 181.1141$, found 181.1137.

Trimethyl(2-methylbenzyl)phosphonium 4-Methylbenzenesulfonate (3d). Following GP1, the compound was formed using trimethyl(2-methylbenzyl)phosphonium bromide $(0.52 \mathrm{~g}, 2 \mathrm{mmol})$, silver $p$-toluenesulfonate $(0.61 \mathrm{~g}, 2.2 \mathrm{mmol}, 1.1 \mathrm{equiv})$, and chloroform $(5 \mathrm{~mL})$. The title compound was obtained as a white solid $(0.57 \mathrm{~g}, 1.6 \mathrm{mmol}, 81 \%):{ }^{1} \mathrm{H}$ NMR $\left(600 \mathrm{MHz}, \mathrm{CDCl}_{3}\right) \delta 7.77$ $(\mathrm{d}, J=8.1 \mathrm{~Hz}, 2 \mathrm{H}), 7.22-7.19(\mathrm{~m}, 3 \mathrm{H}), 7.15-7.14(\mathrm{~m}, 3 \mathrm{H}), 3.95(\mathrm{~d}$, $J=16.4 \mathrm{~Hz}, 2 \mathrm{H}), 2.33(\mathrm{~s}, 3 \mathrm{H}), 2.31(\mathrm{~d}, J=1.3 \mathrm{~Hz}, 3 \mathrm{H}), 2.06(\mathrm{~d}, J=$ $14.3 \mathrm{~Hz}, 9 \mathrm{H}) ;{ }^{13} \mathrm{C}\left\{{ }^{1} \mathrm{H}\right\}$ NMR $\left(151 \mathrm{MHz}, \mathrm{CDCl}_{3}\right) \delta 143.7,139.4$, $137.0\left(\mathrm{~d},{ }^{3} J_{\mathrm{C}-\mathrm{P}}=5.7 \mathrm{~Hz}\right), 131.4\left(\mathrm{~d},{ }^{5} J_{\mathrm{C}-\mathrm{P}}=3.4 \mathrm{~Hz}\right), 131.2\left(\mathrm{~d},{ }^{3} J_{\mathrm{C}-\mathrm{P}}=\right.$ $4.9 \mathrm{~Hz}), 128.7,128.3\left(\mathrm{~d},{ }^{4} J_{\mathrm{C}-\mathrm{P}}=4.0 \mathrm{~Hz}\right), 127.1\left(\mathrm{~d},{ }^{2} J_{\mathrm{C}-\mathrm{P}}=9.0 \mathrm{~Hz}\right)$, $126.7\left(\mathrm{~d},{ }^{4} J_{\mathrm{C}-\mathrm{P}}=3.6 \mathrm{~Hz}\right), 125.8,27.4\left(\mathrm{~d},{ }^{1} J_{\mathrm{C}-\mathrm{P}}=49.1 \mathrm{~Hz}\right), 21.3,20.2$ $\left(\mathrm{d},{ }^{4} J_{\mathrm{C}-\mathrm{P}}=1.4 \mathrm{~Hz}\right), 8.0\left(\mathrm{~d},{ }^{1} J_{\mathrm{C}-\mathrm{P}}=54.0 \mathrm{~Hz}\right) ;{ }^{31} \mathrm{P} \mathrm{NMR}(243 \mathrm{MHz}$, $\left.\mathrm{CDCl}_{3}\right) \delta$ 28.85; HRMS $\mathrm{m} / z$ (ESI+) $\left[\mathrm{M}-\mathrm{OTs}^{+}\right.$calcd for $\left[\mathrm{C}_{11} \mathrm{H}_{18} \mathrm{P}\right]^{+}$181.1141, found 181.1134 .

(3-Fluorobenzyl)trimethylphosphonium Chloride (2e). To a solution of 3-fluorobenzyl chloride $(0.60 \mathrm{~mL}, 5 \mathrm{mmol})$ in acetonitrile $(10 \mathrm{~mL})$ was added a $1.0 \mathrm{M}$ solution of trimethylphosphine in THF $(5.5 \mathrm{~mL}, 5.5 \mathrm{mmol}, 1.1$ equiv) dropwise. The reaction mixture was stirred at $60{ }^{\circ} \mathrm{C}$ for $6 \mathrm{~h}$ and then at room temperature for $16 \mathrm{~h}$, under an argon atmosphere. The solvent was then removed, and the salt precipitated with $\mathrm{Et}_{2} \mathrm{O}$. The salt was collected by filtration, washed with $\mathrm{Et}_{2} \mathrm{O}$, and dried in vacuo to yield the title compound as a white powder (0.92 g, $4.2 \mathrm{mmol}, 84 \%):{ }^{1} \mathrm{H}$ NMR (400 MHz, DMSO- $\left.d_{6}\right) \delta$ $7.50-7.44(\mathrm{~m}, 1 \mathrm{H}), 7.24-7.17(\mathrm{~m}, 3 \mathrm{H}), 3.90(\mathrm{~d}, J=17.2 \mathrm{~Hz}, 2 \mathrm{H})$, $1.83(\mathrm{~d}, J=14.8 \mathrm{~Hz}, 9 \mathrm{H}) ;{ }^{13} \mathrm{C}\left\{{ }^{1} \mathrm{H}\right\}$ NMR (101 MHz, DMSO-d $\left.{ }_{6}\right) \delta$ $162.3\left(\mathrm{dd},{ }^{1} J_{\mathrm{C}-\mathrm{F}}=244.2 \mathrm{~Hz},{ }^{4} J_{\mathrm{C}-\mathrm{P}}=3.9 \mathrm{~Hz}\right), 132.4\left(\mathrm{dd},{ }^{3} J_{\mathrm{C}-\mathrm{F}}=8.5\right.$ $\left.\mathrm{Hz},{ }^{2} J_{\mathrm{C}-\mathrm{P}}=8.5 \mathrm{~Hz}\right), 131.2\left(\mathrm{dd},{ }^{3} J_{\mathrm{C}-\mathrm{F}}=8.9 \mathrm{~Hz},{ }^{4} J_{\mathrm{C}-\mathrm{P}}=3.6 \mathrm{~Hz}\right), 126.2$ $\left(\mathrm{dd},{ }^{4} J_{\mathrm{C}-\mathrm{F}}=3.0 \mathrm{~Hz},{ }^{3} J_{\mathrm{C}-\mathrm{P}}=5.5 \mathrm{~Hz}\right), 116.8\left(\mathrm{dd},{ }^{2} J_{\mathrm{C}-\mathrm{F}}=22.3 \mathrm{~Hz},{ }^{3} J_{\mathrm{C}-\mathrm{P}}\right.$ $=5.2 \mathrm{~Hz}), 114.9\left(\mathrm{dd},{ }^{2} J_{\mathrm{C}-\mathrm{F}}=21.2 \mathrm{~Hz},{ }^{5} J_{\mathrm{C}-\mathrm{P}}=3.9 \mathrm{~Hz}\right), 29.2\left(\mathrm{~d},{ }^{1} J_{\mathrm{C}-\mathrm{P}}\right.$ $=49.1 \mathrm{~Hz}), 7.1\left(\mathrm{~d},{ }^{1} J_{\mathrm{C}-\mathrm{P}}=54.0 \mathrm{~Hz}\right) ;{ }^{19} \mathrm{~F}$ NMR $(376 \mathrm{MHz}$, DMSO$\left.d_{6}\right) \delta-113.08\left(\mathrm{~d},{ }^{5} J_{\mathrm{F}-\mathrm{P}}=2.7 \mathrm{~Hz}\right) ;{ }^{31} \mathrm{P}$ NMR $\left(243 \mathrm{MHz}, \mathrm{DMSO}-d_{6}\right) \delta$ 28.11. HRMS $m / z$ (ESI +$)[\mathrm{M}-\mathrm{Br}]^{+}$calcd for $\left[\mathrm{C}_{10} \mathrm{H}_{15} \mathrm{FP}\right]^{+}$ 185.0890, found 185.0882 .

(3-Fluorobenzyl)trimethylphosphonium 4-Methylbenzenesulfonate (3e). Following GP1, the compound was formed using (3fluorobenzyl)trimethylphosphonium chloride $(0.44 \mathrm{~g}, 2 \mathrm{mmol})$, silver $p$-toluenesulfonate $(0.61 \mathrm{~g}, 2.2 \mathrm{mmol}, 1.1$ equiv), and chloroform (10 $\mathrm{mL}$ ). The mixture was stirred at room temperature for $3 \mathrm{~h}$ rather than $30 \mathrm{~min}$. The title compound was obtained as an off-white solid ( 0.52 g, $1.5 \mathrm{mmol}, 73 \%)$ that turned reddish in color over time: ${ }^{1} \mathrm{H}$ NMR $\left(400 \mathrm{MHz}, \mathrm{DMSO}-d_{6}\right) \delta 7.51(\mathrm{~d}, J=8.1 \mathrm{~Hz}, 2 \mathrm{H}), 7.49-7.43(\mathrm{~m}$, $1 \mathrm{H}), 7.24-7.17(\mathrm{~m}, 3 \mathrm{H}), 7.12(\mathrm{~d}, J=8.0 \mathrm{~Hz}, 2 \mathrm{H}), 3.81(\mathrm{~d}, J=17.1$ $\mathrm{Hz}, 2 \mathrm{H}), 2.29(\mathrm{~s}, 3 \mathrm{H}), 1.80(\mathrm{~d}, J=14.8 \mathrm{~Hz}, 9 \mathrm{H}) ;{ }^{13} \mathrm{C}\left\{{ }^{1} \mathrm{H}\right\} \mathrm{NMR}(101$ $\left.\mathrm{MHz}, \mathrm{DMSO}-d_{6}\right) \delta 162.3\left(\mathrm{dd},{ }^{1} J_{\mathrm{C}-\mathrm{F}}=244.9 \mathrm{~Hz},{ }^{4} J_{\mathrm{C}-\mathrm{P}}=3.8 \mathrm{~Hz}\right)$, $145.7,137.8,132.3\left(\mathrm{dd},{ }^{3} J_{\mathrm{C}-\mathrm{F}}=8.7 \mathrm{~Hz},{ }^{2} \mathrm{~J}_{\mathrm{C}-\mathrm{P}}=8.7 \mathrm{~Hz}\right), 131.2(\mathrm{dd}$, $\left.{ }^{3} J_{\mathrm{C}-\mathrm{F}}=8.7 \mathrm{~Hz},{ }^{4} J_{\mathrm{C}-\mathrm{P}}=3.6 \mathrm{~Hz}\right), 128.2,126.2\left(\mathrm{dd},{ }^{4} J_{\mathrm{C}-\mathrm{F}}=3.0 \mathrm{~Hz}\right.$, $\left.{ }^{3} J_{\mathrm{C}-\mathrm{P}}=5.3 \mathrm{~Hz}\right), 125.6,116.8\left(\mathrm{dd},{ }^{2} J_{\mathrm{C}-\mathrm{F}}=22.2 \mathrm{~Hz},{ }^{3} J_{\mathrm{C}-\mathrm{P}}=5.4 \mathrm{~Hz}\right)$, $115.0\left(\mathrm{dd},{ }^{2} J_{\mathrm{C}-\mathrm{F}}=21.1 \mathrm{~Hz},{ }^{5} J_{\mathrm{C}-\mathrm{P}}=4.0 \mathrm{~Hz}\right), 29.1\left(\mathrm{~d},{ }^{1} J_{\mathrm{C}-\mathrm{P}}=48.9 \mathrm{~Hz}\right)$, 20.9, 7.0 (d, $\left.{ }^{1} J_{\mathrm{C}-\mathrm{P}}=54.0 \mathrm{~Hz}\right) ;{ }^{19} \mathrm{~F}$ NMR $\left(376 \mathrm{MHz}, \mathrm{DMSO}-d_{6}\right) \delta$ $-113.02\left(\mathrm{~d},{ }^{5} J_{\mathrm{F}-\mathrm{P}}=2.7 \mathrm{~Hz}\right) ;{ }^{31} \mathrm{P} \mathrm{NMR}\left(243 \mathrm{MHz}, \mathrm{DMSO}-d_{6}\right) \delta$ 28.07; HRMS $m / z$ (ESI+) $[\mathrm{M}-\mathrm{OTs}]^{+}$calcd for $\left[\mathrm{C}_{10} \mathrm{H}_{15} \mathrm{FP}\right]^{+}$ 185.0890, found 185.0887 .

Benzyltrimethylphosphonium Bromide (2f). To a solution of benzyl bromide $(1.2 \mathrm{~mL}, 10 \mathrm{mmol})$ in acetonitrile $(20 \mathrm{~mL})$ was added a $1.0 \mathrm{M}$ solution of trimethylphosphine in toluene $(11 \mathrm{~mL}, 11 \mathrm{mmol}$, 1.1 equiv) dropwise. The reaction mixture was stirred at room temperature for $1 \mathrm{~h}$ under an argon atmosphere, with the product being observed to precipitate from the solution. The reaction mixture was cooled on ice and then filtered, and the solids were washed with $\mathrm{Et}_{2} \mathrm{O}$ and then dried in vacuo to yield the title compound as a white powder $(2.36 \mathrm{~g}, 9.6 \mathrm{mmol}, 96 \%):{ }^{1} \mathrm{H}$ NMR $\left(600 \mathrm{MHz} \mathrm{CDCl}_{3}\right) \delta$ $7.43-7.41(\mathrm{~m}, 2 \mathrm{H}), 7.38-7.34(\mathrm{~m}, 3 \mathrm{H}), 4.27(\mathrm{~d}, J=16.1 \mathrm{~Hz}, 2 \mathrm{H})$, $2.16(\mathrm{~d}, J=14.1 \mathrm{~Hz}, 9 \mathrm{H}) ;{ }^{13} \mathrm{C}\left\{{ }^{1} \mathrm{H}\right\} \mathrm{NMR}\left(151 \mathrm{MHz}, \mathrm{CDCl}_{3}\right) \delta 130.1$ $\left(\mathrm{d},{ }^{3} J_{\mathrm{C}-\mathrm{P}}=5.4 \mathrm{~Hz}\right), 129.5\left(\mathrm{~d},{ }^{4} J_{\mathrm{C}-\mathrm{P}}=3.5 \mathrm{~Hz}\right), 128.5\left(\mathrm{~d},{ }^{5} J_{\mathrm{C}-\mathrm{P}}=4.0\right.$
$\mathrm{Hz}), 128.2\left(\mathrm{~d},{ }^{2} J_{\mathrm{C}-\mathrm{P}}=9.2 \mathrm{~Hz}\right), 30.6\left(\mathrm{~d},{ }^{1} J_{\mathrm{C}-\mathrm{P}}=49.6 \mathrm{~Hz}\right), 8.5\left(\mathrm{~d},{ }^{1} J_{\mathrm{C}-\mathrm{P}}\right.$ $=54.8 \mathrm{~Hz}) ;{ }^{31} \mathrm{P}$ NMR $\left(243 \mathrm{MHz}, \mathrm{CDCl}_{3}\right) \delta 26.32$. HRMS $\mathrm{m} / z$ (ESI +) $[\mathrm{M}-\mathrm{Br}]^{+}$calcd for $\left[\mathrm{C}_{10} \mathrm{H}_{16} \mathrm{P}\right]^{+}$167.0984, found 167.0978.

Benzyltrimethylphosphonium 4-Methylbenzenesulfonate (3f). Following GP1, the compound was formed using benzyltrimethylphosphonium bromide $(0.99 \mathrm{~g}, 4 \mathrm{mmol})$, silver $p$-toluenesulfonate (1.23 g, $4.4 \mathrm{mmol}, 1.1$ equiv), and chloroform $(10 \mathrm{~mL})$. The mixture was stirred at room temperature for $1.5 \mathrm{~h}$ rather than $30 \mathrm{~min}$. The title compound was obtained as a white solid $(1.32 \mathrm{~g}, 3.9 \mathrm{mmol}, 98 \%):{ }^{1} \mathrm{H}$ NMR $\left(600 \mathrm{MHz}, \mathrm{CDCl}_{3}\right) \delta 7.77(\mathrm{~d}, J=8.1 \mathrm{~Hz}, 2 \mathrm{H}), 7.27-7.26(\mathrm{~m}$, $3 \mathrm{H}), 7.23-7.21(\mathrm{~m}, 2 \mathrm{H}), 7.14(\mathrm{~d}, J=8.0 \mathrm{~Hz}, 2 \mathrm{H}), 3.93(\mathrm{~d}, J=16.4$ $\mathrm{Hz}, 2 \mathrm{H}), 2.32(\mathrm{~s}, 3 \mathrm{H}), 1.95(\mathrm{~d}, J=14.3 \mathrm{~Hz}, 9 \mathrm{H}) ;{ }^{13} \mathrm{C}\left\{{ }^{1} \mathrm{H}\right\} \mathrm{NMR}(151$ $\left.\mathrm{MHz}, \mathrm{CDCl}_{3}\right) \delta 143.8,139.4,130.2\left(\mathrm{~d},{ }^{3} J_{\mathrm{C}-\mathrm{P}}=5.3 \mathrm{~Hz}\right), 129.2(\mathrm{~d}$, $\left.{ }^{4} J_{\mathrm{C}-\mathrm{P}}=3.5 \mathrm{~Hz}\right), 128.81,128.75\left(\mathrm{~d},{ }^{2} J_{\mathrm{C}-\mathrm{P}}=9.2 \mathrm{~Hz}\right), 128.1\left(\mathrm{~d},{ }^{5} J_{\mathrm{C}-\mathrm{P}}=\right.$ $3.9 \mathrm{~Hz}), 125.8,29.9\left(\mathrm{~d},{ }^{1} J_{\mathrm{C}-\mathrm{P}}=49.0 \mathrm{~Hz}\right), 21.3,7.4\left(\mathrm{~d},{ }^{1} \mathrm{~J}_{\mathrm{C}-\mathrm{P}}=54.5\right.$ $\mathrm{Hz}) ;{ }^{31} \mathrm{P}$ NMR (243 $\left.\mathrm{MHz}, \mathrm{CDCl}_{3}\right) \delta 27.16$; HRMS $m / z$ (ESI + ) [M - OTs $]^{+}$calcd for $\left[\mathrm{C}_{10} \mathrm{H}_{16} \mathrm{P}\right]^{+}$167.0984, found 167.0982.

(2-lodobenzyl)trimethylphosphonium Bromide (2g). To a solution of 2-iodobenzyl bromide $(1.48 \mathrm{~g}, 5 \mathrm{mmol})$ in acetonitrile $(10 \mathrm{~mL})$ was added a $1.0 \mathrm{M}$ solution of trimethylphosphine in toluene (11 mL, $11 \mathrm{mmol}, 2.2$ equiv) dropwise. The reaction mixture was stirred at room temperature for $3 \mathrm{~h}$ under an argon atmosphere. The solvent was removed, and the salt precipitated with $\mathrm{CHCl}_{3}$ and $\mathrm{Et}_{2} \mathrm{O}$ (approximately a 1:5 ratio of $\mathrm{CHCl}_{3} / \mathrm{Et}_{2} \mathrm{O}$ ). The salt was collected by filtration, washed with $\mathrm{Et}_{2} \mathrm{O}$, and dried in vacuo to yield the title compound as a white powder $(1.09 \mathrm{~g}, 2.9 \mathrm{mmol}, 58 \%):{ }^{1} \mathrm{H}$ NMR $\left(600 \mathrm{MHz}, \mathrm{CDCl}_{3}\right) \delta 7.90(\mathrm{~d}, J=8.0 \mathrm{~Hz}, 1 \mathrm{H}), 7.74(\mathrm{dt}, J=7.8,2.2$ $\mathrm{Hz}, 1 \mathrm{H}), 7.42(\mathrm{t}, J=7.6 \mathrm{~Hz}, 1 \mathrm{H}), 7.06(\mathrm{tt}, J=7.8,2.0 \mathrm{~Hz}, 1 \mathrm{H}), 4.41$ $(\mathrm{d}, J=16.1 \mathrm{~Hz}, 2 \mathrm{H}), 2.28(\mathrm{~d}, J=14.1 \mathrm{~Hz}, 9 \mathrm{H}) ;{ }^{13} \mathrm{C}\left\{{ }^{1} \mathrm{H}\right\} \operatorname{NMR}(101$ $\left.\mathrm{MHz}, \mathrm{CDCl}_{3}\right) \delta 140.6\left(\mathrm{~d},{ }^{4} J_{\mathrm{C}-\mathrm{P}}=3.2 \mathrm{~Hz}\right), 132.4\left(\mathrm{~d},{ }^{2} J_{\mathrm{C}-\mathrm{P}}=9.2 \mathrm{~Hz}\right)$, $131.8\left(\mathrm{~d},{ }^{3} J_{\mathrm{C}-\mathrm{P}}=4.9 \mathrm{~Hz}\right), 130.4\left(\mathrm{~d},{ }^{4} J_{\mathrm{C}-\mathrm{P}}=3.9 \mathrm{~Hz}\right), 129.5\left(\mathrm{~d},{ }^{5} J_{\mathrm{C}-\mathrm{P}}=\right.$ $3.6 \mathrm{~Hz}), 101.0\left(\mathrm{~d},{ }^{3} J_{\mathrm{C}-\mathrm{P}}=6.9 \mathrm{~Hz}\right), 35.0\left(\mathrm{~d},{ }^{1} J_{\mathrm{C}-\mathrm{P}}=49.9 \mathrm{~Hz}\right), 9.4(\mathrm{~d}$, $\left.{ }^{1} J_{\mathrm{C}-\mathrm{P}}=54.0 \mathrm{~Hz}\right) ;{ }^{31} \mathrm{P}$ NMR $\left(243 \mathrm{MHz}, \mathrm{CDCl}_{3}\right) \delta 28.90$; HRMS $\mathrm{m} / z$ (ESI+) $[\mathrm{M}-\mathrm{Br}]^{+}$calcd for $\left[\mathrm{C}_{10} \mathrm{H}_{15} \mathrm{IP}\right]^{+} 292.9951$, found 292.9940 .

(2-lodobenzyl)trimethylphosphonium 4-Methylbenzenesulfonate $(3 g)$. Following GP1, the compound was formed using (2iodobenzyl)trimethylphosphonium bromide ( $0.75 \mathrm{~g}, 2 \mathrm{mmol})$, silver $p$-toluenesulfonate $(0.61 \mathrm{~g}, 2.2 \mathrm{mmol}, 1.1$ equiv), and chloroform (10 $\mathrm{mL})$. The title compound was obtained as a white solid $(0.90 \mathrm{~g}, 1.9$ mmol, 95\%): ${ }^{1} \mathrm{H}$ NMR $\left(600 \mathrm{MHz}, \mathrm{CDCl}_{3}\right) \delta 7.85(\mathrm{~d}, J=8.0 \mathrm{~Hz}$, $1 \mathrm{H}), 7.75(\mathrm{~d}, J=8.1 \mathrm{~Hz}, 2 \mathrm{H}), 7.53(\mathrm{dt}, J=7.8,2.3 \mathrm{~Hz}, 1 \mathrm{H}), 7.33(\mathrm{t}, J$ $=7.5 \mathrm{~Hz}, 1 \mathrm{H}), 7.13(\mathrm{~d}, J=7.9 \mathrm{~Hz}, 2 \mathrm{H}), 7.00(\mathrm{tt}, J=8.0,1.8 \mathrm{~Hz}, 1 \mathrm{H})$, $4.11(\mathrm{~d}, J=16.2 \mathrm{~Hz}, 2 \mathrm{H}), 2.32(\mathrm{~s}, 3 \mathrm{H}), 2.13(\mathrm{~d}, J=14.4 \mathrm{~Hz}, 9 \mathrm{H})$; ${ }^{13} \mathrm{C}\left\{{ }^{1} \mathrm{H}\right\}$ NMR $\left(151 \mathrm{MHz}, \mathrm{CDCl}_{3}\right) \delta 143.8,140.4\left(\mathrm{~d},{ }^{4} J_{\mathrm{C}-\mathrm{P}}=3.1 \mathrm{~Hz}\right)$, $139.4,132.9\left(\mathrm{~d},{ }^{2} J_{\mathrm{C}-\mathrm{P}}=9.1 \mathrm{~Hz}\right), 131.7\left(\mathrm{~d},{ }^{3} J_{\mathrm{C}-\mathrm{P}}=4.8 \mathrm{~Hz}\right), 130.1(\mathrm{~d}$, $\left.{ }^{4} J_{\mathrm{C}-\mathrm{P}}=3.8 \mathrm{~Hz}\right), 129.4\left(\mathrm{~d},{ }^{5} J_{\mathrm{C}-\mathrm{P}}=3.6 \mathrm{~Hz}\right), 128.7,125.8,100.8(\mathrm{~d}$, $\left.{ }^{3} J_{\mathrm{C}-\mathrm{P}}=6.8 \mathrm{~Hz}\right), 34.8\left(\mathrm{~d},{ }^{1} J_{\mathrm{C}-\mathrm{P}}=49.3 \mathrm{~Hz}\right), 21.3,8.5\left(\mathrm{~d},{ }^{1} J_{\mathrm{C}-\mathrm{P}}=53.7\right.$ $\mathrm{Hz}$ ); ${ }^{31} \mathrm{P} \mathrm{NMR}\left(243 \mathrm{MHz}, \mathrm{CDCl}_{3}\right.$ ) $\delta 29.76$; HRMS $m / z$ (ESI+) [M - OTs $]^{+}$calcd for $\left[\mathrm{C}_{10} \mathrm{H}_{15} \mathrm{IP}\right]^{+} 292.9951$, found 292.9943 .

(3-lodobenzyl)trimethylphosphonium Bromide (2h). To a solution of 3-iodobenzyl bromide $(0.30 \mathrm{~g}, 1 \mathrm{mmol})$ in acetonitrile $(2 \mathrm{~mL})$ was added a $1.0 \mathrm{M}$ solution of trimethylphosphine in toluene (1.1 $\mathrm{mL}, 1.1 \mathrm{mmol}, 1.1$ equiv) dropwise. The reaction mixture was stirred at room temperature for $1.5 \mathrm{~h}$ under an argon atmosphere, with the product being observed to precipitate from solution. The reaction mixture was then filtered, and the solids were washed with $\mathrm{Et}_{2} \mathrm{O}$ and then dried in vacuo to yield the title compound as a white powder (0.36 g, mmol, $0.97 \mathrm{mmol}, 97 \%):{ }^{1} \mathrm{H}$ NMR $(600 \mathrm{MHz}$, $\left.\mathrm{CD}_{3} \mathrm{OD}\right) \delta 7.79-7.77(\mathrm{~m}, 2 \mathrm{H}), 7.39-7.37(\mathrm{~m}, 1 \mathrm{H}), 7.24-7.21(\mathrm{~m}$, $1 \mathrm{H}), 3.76(\mathrm{~d}, J=16.3 \mathrm{~Hz}, 2 \mathrm{H}), 1.87(\mathrm{~d}, J=14.3 \mathrm{~Hz}, 9 \mathrm{H}) ;{ }^{13} \mathrm{C}\left\{{ }^{1} \mathrm{H}\right\}$ NMR $\left(151 \mathrm{MHz}, \mathrm{CD}_{3} \mathrm{OD}\right) \delta 138.5\left(\mathrm{~d},{ }^{3} J_{\mathrm{C}-\mathrm{P}}=5.4 \mathrm{~Hz}\right), 137.4(\mathrm{~d}$, $\left.{ }^{5} J_{\mathrm{C}-\mathrm{P}}=3.9 \mathrm{~Hz}\right), 131.0\left(\mathrm{~d},{ }^{2} J_{\mathrm{C}-\mathrm{P}}=9.0 \mathrm{~Hz}\right), 130.8\left(\mathrm{~d},{ }^{4} J_{\mathrm{C}-\mathrm{P}}=3.5 \mathrm{~Hz}\right)$, $129.0\left(\mathrm{~d},{ }^{3} J_{\mathrm{C}-\mathrm{P}}=5.0 \mathrm{~Hz}\right), 94.3\left(\mathrm{~d},{ }^{4} J_{\mathrm{C}-\mathrm{P}}=4.1 \mathrm{~Hz}\right), 29.2\left(\mathrm{~d},{ }^{1} J_{\mathrm{C}-\mathrm{P}}=\right.$ $49.8 \mathrm{~Hz}), 6.3\left(\mathrm{~d},{ }^{1} J_{\mathrm{C}-\mathrm{P}}=55.2 \mathrm{~Hz}\right) ;{ }^{31} \mathrm{P}$ NMR $\left(243 \mathrm{MHz}, \mathrm{CD}_{3} \mathrm{OD}\right) \delta$ 27.25. HRMS $\mathrm{m} / \mathrm{z}(\mathrm{ESI}+)[\mathrm{M}-\mathrm{Br}]^{+}$calcd for $\left[\mathrm{C}_{10} \mathrm{H}_{15} \mathrm{IP}\right]^{+}$ 292.9951, found 292.9938 .

(3-lodobenzyl)trimethylphosphonium 4-Methylbenzenesulfonate (3h). Following GP1, the compound was formed using (3iodobenzyl)trimethylphosphonium bromide $(0.25 \mathrm{~g}, 0.67 \mathrm{mmol})$, 
silver $p$-toluenesulfonate $(0.22 \mathrm{~g}, 0.8 \mathrm{mmol}, 1.2 \mathrm{equiv})$, and chloroform $(15 \mathrm{~mL})$. The title compound was obtained as a white solid (0.28 g, $0.60 \mathrm{mmol}, 90 \%):{ }^{1} \mathrm{H}$ NMR $\left(400 \mathrm{MHz}\right.$, DMSO- $\left.d_{6}\right) \delta$ 7.73-7.69 (m, 2H), 7.47-7.44 (m, 2H), 7.32-7.29 (m, 1H), $7.21(\mathrm{t}$, $J=7.7 \mathrm{~Hz}, 1 \mathrm{H}), 7.10-7.08(\mathrm{~m}, 2 \mathrm{H}), 3.69(\mathrm{~d}, J=16.9 \mathrm{~Hz}, 2 \mathrm{H}), 2.26$ $(\mathrm{s}, 3 \mathrm{H}), 1.76(\mathrm{~d}, J=14.7 \mathrm{~Hz}, 9 \mathrm{H}) ;{ }^{13} \mathrm{C}\left\{{ }^{1} \mathrm{H}\right\}$ NMR $(101 \mathrm{MHz}$, DMSO- $\left.d_{6}\right) \delta 145.9,138.2\left(\mathrm{~d},{ }^{3} J_{\mathrm{C}-\mathrm{P}}=5.3 \mathrm{~Hz}\right), 137.6,136.7\left(\mathrm{~d},{ }^{5} J_{\mathrm{C}-\mathrm{P}}\right.$ $=3.8 \mathrm{~Hz}), 132.1\left(\mathrm{~d},{ }^{2} J_{\mathrm{C}-\mathrm{P}}=9.0 \mathrm{~Hz}\right), 131.2\left(\mathrm{~d},{ }^{4} J_{\mathrm{C}-\mathrm{P}}=3.3 \mathrm{~Hz}\right), 129.3$ $\left(\mathrm{d},{ }^{3} J_{\mathrm{C}-\mathrm{P}}=5.1 \mathrm{~Hz}\right), 128.1,125.5,95.6\left(\mathrm{~d},{ }^{4} J_{\mathrm{C}-\mathrm{P}}=4.0 \mathrm{~Hz}\right), 28.9(\mathrm{~d}$, $\left.{ }^{1} J_{\mathrm{C}-\mathrm{P}}=48.8 \mathrm{~Hz}\right), 20.8,7.0\left(\mathrm{~d},{ }^{1} J_{\mathrm{C}-\mathrm{P}}=54.0 \mathrm{~Hz}\right) ;{ }^{31} \mathrm{P} \mathrm{NMR}(243 \mathrm{MHz}$, DMSO- $\left.d_{6}\right) \delta$ 27.86; HRMS $\mathrm{m} / z$ (ESI+) $[\mathrm{M}-\mathrm{OTs}]^{+}$calcd for $\left[\mathrm{C}_{10} \mathrm{H}_{15} \mathrm{IP}\right]^{+}$292.9951, found 292.9938 .

(2-Chlorobenzyl)tri-p-tolylphosphonium 4-Methylbenzenesulfonate (3i): A solution of 2-chlorobenzyl bromide $(0.25 \mathrm{~mL}, 2 \mathrm{mmol})$ and tri( $p$-tolyl $)$ phosphine $(0.85 \mathrm{~g}, 2.8 \mathrm{mmol}, 1.4$ equiv) in acetonitrile $(10 \mathrm{~mL})$ was stirred at $50{ }^{\circ} \mathrm{C}$ for $30 \mathrm{~h}$ under an argon atmosphere. The solvent was then removed, and the crude product was used directly in the next step. The crude product and silver $p$ toluenesulfonate $(0.61 \mathrm{~g}, 2.2 \mathrm{mmol}, 1.1$ equiv) were dissolved in chloroform $(10 \mathrm{~mL})$ and then stirred at room temperature for $2 \mathrm{~h}$. Filtration through $\mathrm{MgSO}_{4}$ and removal of the solvent under reduced pressure yielded the crude product as a yellow solid, which darkened to a gray solid overnight and was then purified by flash column chromatography on silica gel $\left(5 \% \mathrm{MeOH}\right.$ in $\left.\mathrm{CH}_{2} \mathrm{Cl}_{2}\right)$. NMR analysis of the product showed that not all of the product was present as the tosylate; thus, the ion exchange reaction was repeated. Filtration through $\mathrm{MgSO}_{4}$ and removal of the solvent under reduced pressure yielded the title compound as a yellow solid $(0.52 \mathrm{~g}, 0.87 \mathrm{mmol}, 44 \%$ over two steps): ${ }^{1} \mathrm{H}$ NMR $\left(600 \mathrm{MHz}, \mathrm{CDCl}_{3}\right) \delta 7.75(\mathrm{~d}, J=8.1 \mathrm{~Hz}$, $2 \mathrm{H}), 7.48(\mathrm{dd}, J=12.5,8.0 \mathrm{~Hz}, 6 \mathrm{H}), 7.44(\mathrm{dt}, J=7.9,2.3 \mathrm{~Hz}, 1 \mathrm{H})$, $7.38(\mathrm{dd}, J=8.1,3.5 \mathrm{~Hz}, 6 \mathrm{H}), 7.20-7.14(\mathrm{~m}, 2 \mathrm{H}), 7.10(\mathrm{t}, J=7.5 \mathrm{~Hz}$, $1 \mathrm{H}), 7.01(\mathrm{~d}, J=7.9 \mathrm{~Hz}, 2 \mathrm{H}), 5.15(\mathrm{~d}, J=14.4 \mathrm{~Hz}, 2 \mathrm{H}), 2.46(\mathrm{~s}, 9 \mathrm{H})$, $2.28(\mathrm{~s}, 3 \mathrm{H}) ;{ }^{13} \mathrm{C}\left\{{ }^{1} \mathrm{H}\right\} \mathrm{NMR}\left(151 \mathrm{MHz}, \mathrm{CDCl}_{3}\right) \delta 146.1\left(\mathrm{~d},{ }^{4} J_{\mathrm{C}-\mathrm{P}}=\right.$ $3.0 \mathrm{~Hz}), 144.6,138.2,135.7\left(\mathrm{~d},{ }^{3} J_{\mathrm{C}-\mathrm{P}}=6.3 \mathrm{~Hz}\right), 134.1\left(\mathrm{~d},{ }^{3} J_{\mathrm{C}-\mathrm{P}}=\right.$ $10.3 \mathrm{~Hz}), 133.4\left(\mathrm{~d},{ }^{3} J_{\mathrm{C}-\mathrm{P}}=4.8 \mathrm{~Hz}\right), 130.8\left(\mathrm{~d},{ }^{2} J_{\mathrm{C}-\mathrm{P}}=13.0 \mathrm{~Hz}\right), 129.7$ $\left(\mathrm{d},{ }^{4} J_{\mathrm{C}-\mathrm{P}}=3.9 \mathrm{~Hz}\right), 129.4\left(\mathrm{~d},{ }^{5} J_{\mathrm{C}-\mathrm{P}}=3.2 \mathrm{~Hz}\right), 128.1,127.7\left(\mathrm{~d},{ }^{4} J_{\mathrm{C}-\mathrm{P}}=\right.$ $3.6 \mathrm{~Hz}), 126.5\left(\mathrm{~d},{ }^{2} J_{\mathrm{C}-\mathrm{P}}=9.1 \mathrm{~Hz}\right), 126.2,114.4\left(\mathrm{~d},{ }^{1} J_{\mathrm{C}-\mathrm{P}}=88.5 \mathrm{~Hz}\right)$, $27.8\left(\mathrm{~d},{ }^{1} \mathrm{~J}_{\mathrm{C}-\mathrm{P}}=50.3 \mathrm{~Hz}\right), 21.8\left(\mathrm{~d},{ }^{5} \mathrm{~J}_{\mathrm{C}-\mathrm{P}}=1.4 \mathrm{~Hz}\right), 21.2 ;{ }^{31} \mathrm{P} \mathrm{NMR}$ $\left(243 \mathrm{MHz}, \mathrm{CDCl}_{3}\right) \delta 21.71$; HRMS $m / z$ (ESI+) $[\mathrm{M}-\mathrm{OTs}]^{+}$calcd for $\left[\mathrm{C}_{28} \mathrm{H}_{27} \mathrm{ClP}\right]^{+} 429.1533$, found 429.1522 .

Trimethyl(pyridin-2-ylmethyl)phosphonium 4-Methylbenzenesulfonate (5a): Powdered potassium hydroxide (0.88 g, 15.68 $\mathrm{mmol}$ ) was added to a vigorously stirred solution of 2pyridinemethanol $(1.0 \mathrm{~mL}, 10.36 \mathrm{mmol})$ in THF $(50 \mathrm{~mL})$ at $0{ }^{\circ} \mathrm{C}$. The reaction mixture was stirred for $15 \mathrm{~min}$; then $p$-toluenesulfonyl chloride $(2.56 \mathrm{~g}, 13.43 \mathrm{mmol})$ was added. The reaction mixture was then stirred for a further $18 \mathrm{~h}$ at room temperature. The reaction mixture was quenched with $\mathrm{NaHCO}_{3}$, and THF was removed under reduced pressure. The product was then extracted with ethyl acetate $(3 \times 40 \mathrm{~mL})$, dried with $\mathrm{MgSO}_{4}$, and concentrated under reduced pressure to give a dark red oil. Purification by flash column chromatography on silica gel (20\% EtOAc in petroleum ether $40-$ $60)$ afforded the title compound as an orange solid (2.11 g, 8.01 mmol, 77\%): ${ }^{1} \mathrm{H}$ NMR $\left(600 \mathrm{MHz}, \mathrm{CDCl}_{3}\right) \delta 8.51(\mathrm{~d}, J=4.6 \mathrm{~Hz}$, $1 \mathrm{H}), 7.83(\mathrm{~d}, J=8.3 \mathrm{~Hz}, 2 \mathrm{H}), 7.70(\mathrm{td}, J=7.9,1.8 \mathrm{~Hz}, 1 \mathrm{H}), 7.42(\mathrm{~d}, J$ $=7.9 \mathrm{~Hz}, 1 \mathrm{H}), 7.34(\mathrm{~d}, J=8.1 \mathrm{~Hz}, 2 \mathrm{H}), 7.23(\mathrm{dd}, J=8.0,5.2 \mathrm{~Hz}$, $1 \mathrm{H}), 5.14(\mathrm{~s}, 2 \mathrm{H}), 2.44(\mathrm{~s}, 3 \mathrm{H}) ;{ }^{13} \mathrm{C}\left\{{ }^{1} \mathrm{H}\right\} \operatorname{NMR}\left(151 \mathrm{MHz} \mathrm{CDCl}_{3}\right) \delta$ 153.7, 149.3, 145.1, 137.0, 132.7, 129.9, 128.1, 123.4, 122.0, 71.7, 21.6. Data are in good agreement with those reported in the literature. $^{9 \mathrm{a}}$ To a solution of pyridin-2-ylmethyl 4-methylbenzenesulfonate $(1.32 \mathrm{~g}, 5.01 \mathrm{mmol})$ in acetonitrile $(10 \mathrm{~mL})$ was added a $1.0 \mathrm{M}$ solution of trimethylphosphine in toluene $(5.5 \mathrm{~mL}, 5.5 \mathrm{mmol}, 1.1$ equiv) dropwise. The reaction mixture was stirred at room temperature for $16 \mathrm{~h}$ under an argon atmosphere. The solvent was removed, and the salt precipitated with $\mathrm{CHCl}_{3}$ and $\mathrm{Et}_{2} \mathrm{O}$ (approximately a 1:5 ratio of $\mathrm{CHCl}_{3} / \mathrm{Et}_{2} \mathrm{O}$ ). This was followed by filtration through a pad of $\mathrm{MgSO}_{4}$ and elution of the salts with $\mathrm{CHCl}_{3}$. Removal of the solvent under reduced pressure and drying in vacuo afforded the crude product as a red oil. This was dissolved in $\mathrm{CH}_{2} \mathrm{Cl}_{2}$ and filtered through an Agilent SampliQ amino cartridge to remove any acids. The solvent was removed under reduced pressure. The precipitation step (with $\mathrm{CHCl}_{3}$ and $\mathrm{Et}_{2} \mathrm{O}$ ) was repeated to afford the title compound as an orange powder (1.43 g, $4.21 \mathrm{mmol}, 84 \%)$; note that the purification step to remove acid is essential, and the borylation reaction does not work in the presence of traces of acid: ${ }^{1} \mathrm{H}$ $\operatorname{NMR}\left(600 \mathrm{MHz}, \mathrm{CDCl}_{3}\right) \delta 8.48(\mathrm{~d}, J=4.3 \mathrm{~Hz}, 1 \mathrm{H}), 7.78(\mathrm{~d}, J=8.2$ $\mathrm{Hz}, 2 \mathrm{H}), 7.66(\mathrm{td}, J=8.0,1.2 \mathrm{~Hz}, 1 \mathrm{H}), 7.54(\mathrm{~d}, J=7.8 \mathrm{~Hz}, 1 \mathrm{H})$, $7.23-7.21(\mathrm{~m}, 1 \mathrm{H}), 7.14(\mathrm{~d}, J=7.9 \mathrm{~Hz}, 2 \mathrm{H}), 4.15(\mathrm{~d}, J=15.8 \mathrm{~Hz}$, $2 \mathrm{H}), 2.33(\mathrm{~s}, 3 \mathrm{H}), 2.13(\mathrm{~d}, J=14.5 \mathrm{~Hz}, 9 \mathrm{H}) ;{ }^{13} \mathrm{C}\left\{{ }^{1} \mathrm{H}\right\} \operatorname{NMR}(151$ $\left.\mathrm{MHz}, \mathrm{CDCl}_{3}\right) \delta 151.1\left(\mathrm{~d},{ }^{2} J_{\mathrm{C}-\mathrm{P}}=9.4 \mathrm{~Hz}\right), 149.3\left(\mathrm{~d},{ }^{4} J_{\mathrm{C}-\mathrm{P}}=1.9 \mathrm{~Hz}\right)$, 143.9, 139.2, 137.6, 128.6, 125.9, $125.7\left(\mathrm{~d},{ }^{3} J_{\mathrm{C}-\mathrm{P}}=7.3 \mathrm{~Hz}\right), 122.8(\mathrm{~d}$, $\left.{ }^{5} J_{\mathrm{C}-\mathrm{P}}=2.3 \mathrm{~Hz}\right), 32.2\left(\mathrm{~d},{ }^{1} J_{\mathrm{C}-\mathrm{P}}=53.1 \mathrm{~Hz}\right), 21.3,8.9\left(\mathrm{~d},{ }^{1} J_{\mathrm{C}-\mathrm{P}}=54.9\right.$ $\mathrm{Hz}) ;{ }^{31} \mathrm{P} \mathrm{NMR}\left(243 \mathrm{MHz}, \mathrm{CDCl}_{3}\right) \delta 27.46$; HRMS $m / z$ (ESI+) [M - OTs $]^{+}$calcd for $\left[\mathrm{C}_{9} \mathrm{H}_{15} \mathrm{NP}\right]^{+}$168.0937, found 168.0932 .

Trimethyl(2-(trifluoromethyl)phenethyl)phosphonium 4-Methylbenzenesulfonate (7a). 2-(Trifluoromethyl)phenethyl 4-methylbenzenesulfonate was synthesized according to a published procedure as a colorless oil (3.33 g, $7.94 \mathrm{mmol}, 53 \%){ }^{9 \mathrm{~b}}$ 2-(Trifluoromethyl)phenethyl 4-methylbenzenesulfonate $(3.33 \mathrm{~g}, 7.94 \mathrm{mmol})$ was added to a microwave vial, and the vial was sealed, evacuated, and backfilled with argon. Acetonitrile (1 M) was added, followed by trimethylphosphine ( $9.5 \mathrm{~mL}, 1.2$ equiv, $1 \mathrm{M}$ solution in toluene). The resulting reaction mixture was stirred at $80{ }^{\circ} \mathrm{C}$ for $24 \mathrm{~h}$. The solvent was removed under reduced pressure; then $\mathrm{Et}_{2} \mathrm{O}$ was added, and the title phosphonium salt was isolated by filtration as a white solid (2.90 g, $6.91 \mathrm{mmol}, 87 \%):{ }^{1} \mathrm{H}$ NMR $\left(600 \mathrm{MHz}, \mathrm{CD}_{3} \mathrm{OD}\right) \delta 7.68-7.70(\mathrm{~m}$, $3 \mathrm{H}), 7.61(\mathrm{t}, J=7.6 \mathrm{~Hz}, 1 \mathrm{H}), 7.56(\mathrm{~d}, J=7.6 \mathrm{~Hz}, 1 \mathrm{H}), 7.46(\mathrm{t}, J=7.6$ $\mathrm{Hz}, 1 \mathrm{H}), 7.21(\mathrm{~d}, J=8.0 \mathrm{~Hz}, 2 \mathrm{H}), 3.04-3.10(\mathrm{~m}, 2 \mathrm{H}), 2.49-2.54(\mathrm{~m}$, $2 \mathrm{H}), 2.35(\mathrm{~s}, 3 \mathrm{H}), 1.94\left(\mathrm{~d},{ }^{2} \mathrm{~J}_{\mathrm{P}-\mathrm{H}}=14.6 \mathrm{~Hz}, 9 \mathrm{H}\right) ;{ }^{13} \mathrm{C}\left\{{ }^{1} \mathrm{H}\right\} \mathrm{NMR}$ $\left(151 \mathrm{MHz}, \mathrm{CD}_{3} \mathrm{OD}\right) \delta 142.3,140.2,137.9$ (dd, $\left.J=17.4,1.5 \mathrm{~Hz}\right)$, $132.6,131.2,128.4,127.6\left(\mathrm{q},{ }^{2} J_{\mathrm{C}-\mathrm{F}}=29.5 \mathrm{~Hz}\right), 127.2,125.8\left(\mathrm{q},{ }^{3} J_{\mathrm{C}-\mathrm{F}}\right.$ $=5.7 \mathrm{~Hz}), 125.5,124.7\left(\mathrm{q},{ }^{1} J_{\mathrm{C}-\mathrm{F}}=272.9 \mathrm{~Hz}\right), 24.8\left(\mathrm{~d},{ }^{1} J_{\mathrm{C}-\mathrm{P}}=50.6\right.$ $\mathrm{Hz}), 23.9\left(\mathrm{~d},{ }^{2} \mathrm{~J}_{\mathrm{C}-\mathrm{P}}=2.0 \mathrm{~Hz}\right), 19.9,6.2\left(\mathrm{~d},{ }^{1} J_{\mathrm{C}-\mathrm{P}}=54.6 \mathrm{~Hz}\right) ;{ }^{31} \mathrm{P}$ NMR $\left(243 \mathrm{MHz}, \mathrm{CD}_{3} \mathrm{OD}\right) \delta$ 27.33; HRMS (ESI+) calcd for $\left[\mathrm{C}_{12} \mathrm{H}_{17} \mathrm{PF}_{3}\right]^{+}$249.1014, found 249.1020.

General Procedure for Iridium-Catalyzed Borylation (GP2). The reactions were carried out in $4 \mathrm{~mL} 15 \mathrm{~mm} \times 45 \mathrm{~mm}$ crimp top vials. The substrate $(0.25 \mathrm{mmol})$, ligand $1(3 \mathrm{~mol} \%), \mathrm{B}_{2} \operatorname{Pin}_{2}(1.5$ equiv), and $[\operatorname{Ir}(\mathrm{COD}) \mathrm{OMe}]_{2}(1.5 \mathrm{~mol} \%)$ were weighed and added to the vial, which was then sealed, evacuated, and backfilled with argon. 1,4-Dioxane was then added for a final substrate concentration of $0.2 \mathrm{M}$. The reaction mixture was stirred and heated in deep-welled heating blocks (IKA DB 5.2) for a specified amount of time, at a specified temperature, followed by removal of the solvent and analysis of the crude reaction mixture by ${ }^{1} \mathrm{H}$ NMR. Purification was generally performed by addition of $\mathrm{Et}_{2} \mathrm{O}$ to a concentrated $\mathrm{CH}_{2} \mathrm{Cl}_{2}$ solution of the crude reaction mixture, followed by filtration of the resulting precipitate.

Calculation of the Yield in Borylation Reactions. In some cases, small amounts of the starting material remained in the reactions, which were inseparable from the borylated products. The following procedure was then used to determine the yield of the borylated products. The ratio of borylated products to starting material was determined by NMR analysis, using the NMR of the isolated product. This ratio was used to calculate an average molecular weight in order to determine the mmol of product obtained, such that an overall yield could be obtained. The yield of the borylated products was then obtained by multiplying the overall yield by the fraction of borylated products present.

Assignment of meta and para Products. When possible, the coupling patterns in the aromatic region were used to assign the respective isomers. Otherwise, assignments were done using information from 2D NMR experiments (COSY, HSQC, HMBC, NOESY). Data for the para product was usually obtained from the tmphen control experiments by subtracting the signals for the meta product and starting material from the spectra.

Trimethyl(5-(4,4,5,5-tetramethyl-1,3,2-dioxaborolan-2-yl)-2(trifluoromethyl)benzyl)phosphonium 4-Methylbenzenesulfonate (4a). With Sulfonate Ligand 1 (0.1 mmol Scale). Following GP2, the compound was formed using trimethyl(2-(trifluoromethyl)- 
benzyl)phosphonium 4-methylbenzenesulfonate (3a) (40.8 mg, 0.1 $\mathrm{mmol}$ ), $\mathrm{B}_{2} \mathrm{Pin}_{2}$ (38 mg, $0.15 \mathrm{mmol}, 1.5$ equiv), [ $\left.\mathrm{Ir}(\mathrm{COD}) \mathrm{OMe}\right]_{2}(1$ $\mathrm{mg}, 0.0015 \mathrm{mmol}, 0.015$ equiv), 1 ( $1.5 \mathrm{mg}, 0.003 \mathrm{mmol}, 0.03$ equiv), and 1,4-dioxane $(0.5 \mathrm{~mL})$. The reaction mixture was stirred for $16 \mathrm{~h}$ at $50{ }^{\circ} \mathrm{C}$. Analysis of the crude ${ }^{1} \mathrm{H}$ NMR with 1,2-dimethoxyethane as the internal standard showed >20:1 meta/para borylation, in $89 \%$ NMR yield.

With Sulfonate Ligand $1(0.25 \mathrm{mmol}$ Scale). Following GP2, the compound was formed using trimethyl(2-(trifluoromethyl)benzyl)phosphonium 4-methylbenzenesulfonate (3a) $(102 \mathrm{mg}, 0.25 \mathrm{mmol})$, $\mathrm{B}_{2} \mathrm{Pin}_{2}$ (95 mg, $0.375 \mathrm{mmol}, 1.5$ equiv), $[\mathrm{Ir}(\mathrm{COD}) \mathrm{OMe}]_{2}(2.5 \mathrm{mg}$, $0.00375 \mathrm{mmol}, 0.015$ equiv), 1 ( $3.8 \mathrm{mg}, 0.0075 \mathrm{mmol}, 0.03$ equiv), and 1,4-dioxane $(1.25 \mathrm{~mL})$. The reaction mixture was stirred for $16 \mathrm{~h}$ at $50{ }^{\circ} \mathrm{C}$. Analysis of the crude ${ }^{1} \mathrm{H}$ NMR with 1,2-dimethoxyethane as the internal standard showed 6.5:1 meta/para borylation, in 93\% NMR yield. The meta product decomposed upon attempted isolation by precipitation with $\mathrm{Et}_{2} \mathrm{O}$; therefor, the crude NMR data was used in order to characterize the meta product.

Compound 4a: ${ }^{1} \mathrm{H}$ NMR $\left(400 \mathrm{MHz}, \mathrm{CDCl}_{3}\right) \delta 7.82(\mathrm{~d}, J=7.6$ $\mathrm{Hz}, 1 \mathrm{H}), 7.72(\mathrm{~d}, J=8.0 \mathrm{~Hz}, 2 \mathrm{H}), 7.72(\mathrm{~s}, 1 \mathrm{H}), 7.65(\mathrm{~d}, J=7.8 \mathrm{~Hz}$, $1 \mathrm{H}), 7.06(\mathrm{~d}, J=7.9 \mathrm{~Hz}, 2 \mathrm{H}), 3.90(\mathrm{~d}, J=16.4 \mathrm{~Hz}, 2 \mathrm{H}), 2.25(\mathrm{~s}, 3 \mathrm{H})$, $2.04(\mathrm{~d}, J=14.4 \mathrm{~Hz}, 9 \mathrm{H}), 1.31(\mathrm{~s}, 12 \mathrm{H}) ;{ }^{13} \mathrm{C}\left\{{ }^{1} \mathrm{H}\right\}$ NMR $(101 \mathrm{MHz}$, $\left.\mathrm{CDCl}_{3}\right) \delta 143.6,139.2,137.8\left(\mathrm{~d},{ }^{3} J_{\mathrm{C}-\mathrm{P}}=4.8 \mathrm{~Hz}\right), 134.8\left(\mathrm{~d},{ }^{5} J_{\mathrm{C}-\mathrm{P}}=\right.$ $3.3 \mathrm{~Hz}), 130.9\left(\mathrm{qd},{ }^{2} J_{\mathrm{C}-\mathrm{F}}=29.8 \mathrm{~Hz},{ }^{3} J_{\mathrm{C}-\mathrm{P}}=5.9 \mathrm{~Hz}\right), 128.6,126.9(\mathrm{~d}$, $\left.{ }^{2} J_{\mathrm{C}-\mathrm{P}}=8.3 \mathrm{~Hz}\right), 126.4(\mathrm{~m}), 125.9,123.8\left(\mathrm{q},{ }^{1} J_{\mathrm{C}-\mathrm{F}}=273.8 \mathrm{~Hz}\right), 84.7$, $28.0\left(\mathrm{~d},{ }^{1} J_{\mathrm{C}-\mathrm{P}}=49.6 \mathrm{~Hz}\right), 24.8,21.2,8.4\left(\mathrm{~d},{ }^{1} \mathrm{~J}_{\mathrm{C}-\mathrm{P}}=54.1 \mathrm{~Hz}\right) ;{ }^{19} \mathrm{~F}$ $\operatorname{NMR}\left(376 \mathrm{MHz}, \mathrm{CDCl}_{3}\right) \delta-58.43 ;{ }^{31} \mathrm{P} \operatorname{NMR}\left(162 \mathrm{MHz} \mathrm{CDCl}_{3}\right) \delta$ 29.13; HRMS $m / z$ (ESI+) $[\mathrm{M}-\mathrm{OTs}]^{+}$calcd for $\left[\mathrm{C}_{17} \mathrm{H}_{26} \mathrm{BF}_{3} \mathrm{O}_{2} \mathrm{P}\right]^{+}$ 361.1710, found 361.1706 .

With tmphen $(0.25 \mathrm{mmol}$ Scale). Following GP2, the compound was formed using trimethyl(2-(trifluoromethyl)benzyl)phosphonium 4-methylbenzenesulfonate (3a) (102 mg, $0.25 \mathrm{mmol}$ ), $\mathrm{B}_{2} \operatorname{Pin}_{2}$ ( $95 \mathrm{mg}$, $0.375 \mathrm{mmol}, 1.5$ equiv), [ $\operatorname{Ir}(\mathrm{COD}) \mathrm{OMe}]_{2}(2.5 \mathrm{mg}, 0.00375 \mathrm{mmol}$, 0.015 equiv), tmphen $(1.8 \mathrm{mg}, 0.0075 \mathrm{mmol}, 0.03$ equiv), and 1,4dioxane $(1.25 \mathrm{~mL})$. The reaction mixture was stirred for $16 \mathrm{~h}$ at 50 ${ }^{\circ} \mathrm{C}$. Analysis of the crude ${ }^{1} \mathrm{H}$ NMR with 1,2-dimethoxyethane as the internal standard showed 1:1.6 meta/para borylation, in 90\% NMR yield. It was observed that it was possible to isolate the para product by precipitation with $\mathrm{Et}_{2} \mathrm{O}$, while the meta product decomposed.

para Product: ${ }^{1} \mathrm{H}$ NMR $\left(400 \mathrm{MHz}, \mathrm{CDCl}_{3}\right) \delta 8.05(\mathrm{~s}, 1 \mathrm{H}), 7.88$ $(\mathrm{d}, J=7.7 \mathrm{~Hz}, 1 \mathrm{H}), 7.70(\mathrm{~d}, J=8.2 \mathrm{~Hz}, 2 \mathrm{H}), 7.55(\mathrm{dd}, J=7.7,2.5 \mathrm{~Hz}$, $1 \mathrm{H}), 7.06(\mathrm{~d}, J=7.9 \mathrm{~Hz}, 2 \mathrm{H}), 4.01(\mathrm{~d}, J=17.0 \mathrm{~Hz}, 2 \mathrm{H}), 2.25(\mathrm{~s}, 3 \mathrm{H})$, $2.02(\mathrm{~d}, J=14.4 \mathrm{~Hz}, 9 \mathrm{H}), 1.30(\mathrm{~s}, 12 \mathrm{H}) ;{ }^{13} \mathrm{C}\left\{{ }^{1} \mathrm{H}\right\} \mathrm{NMR}(101 \mathrm{MHz}$, $\left.\mathrm{CDCl}_{3}\right) \delta 143.5,139.3,138.9$ (br s), $133.0(\mathrm{~m}), 132.3\left(\mathrm{~d},{ }^{3} J_{\mathrm{C}-\mathrm{P}}=4.8\right.$ $\mathrm{Hz}), 130.2\left(\mathrm{~d},{ }^{2} J_{\mathrm{C}-\mathrm{P}}=9.0 \mathrm{~Hz}\right), 128.6,128.2\left(\mathrm{dq},{ }^{2} J_{\mathrm{C}-\mathrm{F}}=29.5 \mathrm{~Hz}\right.$, $\left.{ }^{3} J_{\mathrm{C}-\mathrm{P}}=5.6 \mathrm{~Hz}\right), 125.8,124.1\left(\mathrm{q},{ }^{1} \mathrm{~J}_{\mathrm{C}-\mathrm{F}}=273.9 \mathrm{~Hz}\right), 84.5,27.9(\mathrm{~d}$, $\left.{ }^{1} J_{\mathrm{C}-\mathrm{P}}=49.4 \mathrm{~Hz}\right), 24.8,21.2,8.3\left(\mathrm{~d},{ }^{1} J_{\mathrm{C}-\mathrm{P}}=54.1 \mathrm{~Hz}\right) ;{ }^{19} \mathrm{~F}$ NMR $(376$ $\left.\mathrm{MHz} \mathrm{CDCl}_{3}\right) \delta-57.75 ;{ }^{31} \mathrm{P}$ NMR $\left(162 \mathrm{MHz}, \mathrm{CDCl}_{3}\right) \delta 29.19$.

(2-Chloro-5-(4,4,5,5-tetramethyl-1,3,2-dioxaborolan-2-yl)benzyl)trimethylphosphonium 4-Methylbenzenesulfonate (4b). With Sulfonate Ligand 1. Following GP2, the compound was formed using (2-chlorobenzyl)trimethylphosphonium 4-methylbenzenesulfonate ( $3 \mathbf{b})(93 \mathrm{mg}, 0.25 \mathrm{mmol}), \mathrm{B}_{2} \mathrm{Pin}_{2}(95 \mathrm{mg}, 0.375 \mathrm{mmol}$, 1.5 equiv), $[\operatorname{Ir}(\mathrm{COD}) \mathrm{OMe}]_{2}(2.5 \mathrm{mg}, 0.00375 \mathrm{mmol}, 0.015$ equiv $), 1$ $(3.8 \mathrm{mg}, 0.0075 \mathrm{mmol}, 0.03$ equiv), and 1,4 -dioxane $(1.25 \mathrm{~mL})$. The reaction mixture was stirred for $16 \mathrm{~h}$ at $50{ }^{\circ} \mathrm{C}$. Analysis of crude ${ }^{1} \mathrm{H}$ NMR showed >20:1 meta/para borylation. The solvent was removed, and the salts precipitated with $\mathrm{Et}_{2} \mathrm{O}$. This was followed by filtration through a pad of $\mathrm{MgSO}_{4}$ and elution of the salts with $\mathrm{CH}_{2} \mathrm{Cl}_{2}$. Removal of the solvent under reduced pressure and drying in vacuo afforded the title compounds (83:3:14 meta/para/SM) as a light orange powder (99 mg, $0.18 \mathrm{mmol}$ borylated products, 70\%, >20:1 meta/para).

Compound $4 \boldsymbol{b}:{ }^{1} \mathrm{H}$ NMR $\left(600 \mathrm{MHz}, \mathrm{CDCl}_{3}\right) \delta 7.79(\mathrm{~d}, J=8.1$ $\mathrm{Hz}, 2 \mathrm{H}), 7.72-7.70(\mathrm{~m}, 2 \mathrm{H}), 7.44(\mathrm{~d}, J=7.8 \mathrm{~Hz}, 1 \mathrm{H}), 7.14(\mathrm{~d}, J=$ $8.0 \mathrm{~Hz}, 2 \mathrm{H}), 3.97(\mathrm{~d}, J=16.0 \mathrm{~Hz}, 2 \mathrm{H}), 2.32(\mathrm{~s}, 3 \mathrm{H}), 2.13(\mathrm{~d}, J=14.3$ $\mathrm{Hz}, 9 \mathrm{H}), 1.34(\mathrm{~s}, 12 \mathrm{H}) ;{ }^{13} \mathrm{C}\left\{{ }^{1} \mathrm{H}\right\}$ NMR $\left(101 \mathrm{MHz}, \mathrm{CDCl}_{3}\right) \delta 143.7$, $139.3,137.9\left(\mathrm{~d},{ }^{3} J_{\mathrm{C}-\mathrm{P}}=4.8 \mathrm{~Hz}\right), 137.1\left(\mathrm{~d},{ }^{3} J_{\mathrm{C}-\mathrm{P}}=6.0 \mathrm{~Hz}\right), 136.3(\mathrm{~d}$, $\left.{ }^{5} J_{\mathrm{C}-\mathrm{P}}=3.8 \mathrm{~Hz}\right), 129.9\left(\mathrm{~d},{ }^{4} J_{\mathrm{C}-\mathrm{P}}=3.0 \mathrm{~Hz}\right), 128.7,126.5\left(\mathrm{~d},{ }^{2} J_{\mathrm{C}-\mathrm{P}}=9.1\right.$ $\mathrm{Hz}), 125.9,84.5,28.4\left(\mathrm{~d},{ }^{1} J_{\mathrm{C}-\mathrm{P}}=49.8 \mathrm{~Hz}\right), 24.9,21.3,8.4\left(\mathrm{~d},{ }^{1} J_{\mathrm{C}-\mathrm{P}}=\right.$
$54.0 \mathrm{~Hz}$ ); ${ }^{31} \mathrm{P}$ NMR $\left(243 \mathrm{MHz}, \mathrm{CDCl}_{3}\right) \delta 29.17$; HRMS $m / z$ (ESI+) $[\mathrm{M}-\mathrm{OTs}]^{+}$calcd for $\left[\mathrm{C}_{16} \mathrm{H}_{26} \mathrm{BClO}_{2} \mathrm{P}\right]^{+} 327.1447$, found 327.1443 .

With tmphen. Following GP2, the compound was formed using (2-chlorobenzyl)trimethylphosphonium 4-methylbenzenesulfonate (3b) (93 mg, $0.25 \mathrm{mmol}$ ), $\mathrm{B}_{2} \mathrm{Pin}_{2}$ (95 mg, $0.375 \mathrm{mmol}, 1.5$ equiv), $[\operatorname{Ir}(\mathrm{COD}) \mathrm{OMe}]_{2}(2.5 \mathrm{mg}, 0.00375 \mathrm{mmol}, 0.015$ equiv $)$, tmphen ( 1.8 $\mathrm{mg}, 0.0075 \mathrm{mmol}, 0.03$ equiv), and 1,4-dioxane. The reaction mixture was stirred for $16 \mathrm{~h}$ at $50{ }^{\circ} \mathrm{C}$. Analysis of crude ${ }^{1} \mathrm{H}$ NMR showed a 1:3.3:1.5 ratio of meta/para/starting material. The solvent was removed, and the salts precipitated with $\mathrm{Et}_{2} \mathrm{O}$. This was followed by filtration through a pad of $\mathrm{MgSO}_{4}$ and elution of the salts with $\mathrm{CH}_{2} \mathrm{Cl}_{2}$. Removal of the solvent under reduced pressure and drying in vacuo afforded the title compounds (15:50:35 meta/para/SM) as a brown solid (108 $\mathrm{mg}, 0.15 \mathrm{mmol}$ borylated products, $62 \%, 1: 3.3$ meta/para).

para Product: ${ }^{1} \mathrm{H}$ NMR $\left(600 \mathrm{MHz}, \mathrm{CDCl}_{3}\right) \delta 7.81(\mathrm{~s}, 1 \mathrm{H}), 7.75$ $(\mathrm{d}, J=8.0 \mathrm{~Hz}, 2 \mathrm{H}), 7.62(\mathrm{~d}, J=7.5 \mathrm{~Hz}, 1 \mathrm{H}), 7.48-7.47(\mathrm{~m}, 1 \mathrm{H})$, $7.12(\mathrm{~d}, J=8.0 \mathrm{~Hz}, 2 \mathrm{H}), 4.06(\mathrm{~d}, J=16.7 \mathrm{~Hz}, 2 \mathrm{H}), 2.32(\mathrm{~s}, 3 \mathrm{H}), 2.07$ $(\mathrm{d}, J=14.5 \mathrm{~Hz}, 9 \mathrm{H}), 1.34(\mathrm{~s}, 12 \mathrm{H}) ;{ }^{13} \mathrm{C}\left\{{ }^{1} \mathrm{H}\right\}$ NMR $(101 \mathrm{MHz}$, $\left.\mathrm{CDCl}_{3}\right) \delta 143.7,139.4,136.2\left(\mathrm{~d},{ }^{4} J_{\mathrm{C}-\mathrm{P}}=3.1 \mathrm{~Hz}\right), 133.9\left(\mathrm{~d},{ }^{3} J_{\mathrm{C}-\mathrm{P}}=\right.$ $6.3 \mathrm{~Hz}), 133.7\left(\mathrm{~d},{ }^{3} J_{\mathrm{C}-\mathrm{P}}=6.1 \mathrm{~Hz}\right), 132.1\left(\mathrm{~d},{ }^{4} J_{\mathrm{C}-\mathrm{P}}=4.9 \mathrm{~Hz}\right), 130.0$ $\left(\mathrm{d},{ }^{2} J_{\mathrm{C}-\mathrm{P}}=9.3 \mathrm{~Hz}\right), 128.7,125.8,84.4,28.2\left(\mathrm{~d},{ }^{1} J_{\mathrm{C}-\mathrm{P}}=49.4 \mathrm{~Hz}\right), 24.9$, 21.3, $8.3\left(\mathrm{~d},{ }^{1} J_{\mathrm{C}-\mathrm{P}}=53.8 \mathrm{~Hz}\right) ;{ }^{31} \mathrm{P}$ NMR $\left(243 \mathrm{MHz}, \mathrm{CDCl}_{3}\right) \delta 29.54$.

(2-Bromo-5-(4,4,5,5-tetramethyl-1,3,2-dioxaborolan-2-yl)benzyl)trimethylphosphonium 4-Methylbenzenesulfonate (4c). With Sulfonate Ligand 1. Following GP2, the compound was formed using (2-bromobenzyl)trimethylphosphonium 4-methylbenzenesulfonate (3c) $(104 \mathrm{mg}, 0.25 \mathrm{mmol}), \mathrm{B}_{2} \operatorname{Pin}_{2}(95 \mathrm{mg}, 0.375$ mmol, 1.5 equiv), $[\mathrm{Ir}(\mathrm{COD}) \mathrm{OMe}]_{2}(2.5 \mathrm{mg}, 0.00375 \mathrm{mmol}, 0.015$ equiv), 1 ( $3.8 \mathrm{mg}, 0.0075 \mathrm{mmol}, 0.03$ equiv), and 1,4-dioxane ( 1.25 $\mathrm{mL}$ ). The reaction mixture was stirred for $16 \mathrm{~h}$ at $50{ }^{\circ} \mathrm{C}$. Analysis of crude ${ }^{1} \mathrm{H}$ NMR showed $>20: 1$ meta/para borylation. The solvent was removed, and the salts precipitated with $\mathrm{Et}_{2} \mathrm{O}$. This was followed by filtration through a pad of $\mathrm{MgSO}_{4}$ and elution of the salts with $\mathrm{CH}_{2} \mathrm{Cl}_{2}$. Removal of the solvent under reduced pressure and drying in vacuo afforded the title compounds (85:5:10 meta/para/SM) as a foamy orange-brown solid $(107 \mathrm{mg}, 0.18 \mathrm{mmol}$ borylated products, 73\%, 17:1 meta/para).

Compound 4c: ${ }^{1} \mathrm{H}$ NMR $\left(600 \mathrm{MHz}, \mathrm{CDCl}_{3}\right) \delta 7.78(\mathrm{~d}, J=8.1 \mathrm{~Hz}$, $2 \mathrm{H}), 7.69(\mathrm{~d}, J=1.6 \mathrm{~Hz}, 1 \mathrm{H}), 7.62-7.57(\mathrm{~m}, 2 \mathrm{H}), 7.13(\mathrm{~d}, J=7.9$ $\mathrm{Hz}, 2 \mathrm{H}), 3.98(\mathrm{~d}, J=15.9 \mathrm{~Hz}, 2 \mathrm{H}), 2.31(\mathrm{~s}, 3 \mathrm{H}), 2.12(\mathrm{~d}, J=14.3 \mathrm{~Hz}$, 9H), $1.34(\mathrm{~s}, 12 \mathrm{H}) ;{ }^{13} \mathrm{C}\left\{{ }^{1} \mathrm{H}\right\}$ NMR $\left(101 \mathrm{MHz}, \mathrm{CDCl}_{3}\right) \delta$ 143.7, $139.3,137.7\left(\mathrm{~d},{ }^{3} J_{\mathrm{C}-\mathrm{P}}=4.9 \mathrm{~Hz}\right), 136.3\left(\mathrm{~d},{ }^{5} J_{\mathrm{C}-\mathrm{P}}=3.3 \mathrm{~Hz}\right), 133.3(\mathrm{~d}$, $\left.{ }^{4} J_{\mathrm{C}-\mathrm{P}}=3.2 \mathrm{~Hz}\right), 128.7,128.6\left(\mathrm{~d},{ }^{2} J_{\mathrm{C}-\mathrm{P}}=9.0 \mathrm{~Hz}\right), 128.2\left(\mathrm{~d},{ }^{3} J_{\mathrm{C}-\mathrm{P}}=6.3\right.$ $\mathrm{Hz}), 125.9,84.5,30.9\left(\mathrm{~d},{ }^{1} J_{\mathrm{C}-\mathrm{P}}=49.4 \mathrm{~Hz}\right), 24.9,21.3,8.5\left(\mathrm{~d},{ }^{1} J_{\mathrm{C}-\mathrm{P}}=\right.$ $53.8 \mathrm{~Hz}$ ); ${ }^{31} \mathrm{P}$ NMR $\left(243 \mathrm{MHz}, \mathrm{CDCl}_{3}\right) \delta 29.02 ; \mathrm{HRMS} \mathrm{m} / z$ (ESI+) $[\mathrm{M}-\mathrm{OTs}]^{+}$calcd for $\left[\mathrm{C}_{16} \mathrm{H}_{26} \mathrm{BBrO}_{2} \mathrm{P}\right]^{+}$371.0941, found 371.0931.

With tmphen. Following GP2, the compound was formed using (2-bromobenzyl)trimethylphosphonium 4-methylbenzenesulfonate (3c) (104 mg, $0.25 \mathrm{mmol}), \mathrm{B}_{2} \mathrm{Pin}_{2}(95 \mathrm{mg}, 0.375 \mathrm{mmol}, 1.5$ equiv), $[\operatorname{Ir}(\mathrm{COD}) \mathrm{OMe}]_{2}(2.5 \mathrm{mg}, 0.00375 \mathrm{mmol}, 0.015$ equiv $)$, tmphen (1.8 $\mathrm{mg}, 0.0075 \mathrm{mmol}, 0.03$ equiv), and 1,4-dioxane (1.25 $\mathrm{mL})$. The reaction mixture was stirred for $16 \mathrm{~h}$ at $50{ }^{\circ} \mathrm{C}$. Analysis of crude ${ }^{1} \mathrm{H}$ NMR showed 1:3.5 meta/para borylation. The solvent was removed, and the salts precipitated with $\mathrm{Et}_{2} \mathrm{O}$. This was followed by filtration through a pad of $\mathrm{MgSO}_{4}$ and elution of the salts with $\mathrm{CH}_{2} \mathrm{Cl}_{2}$. Removal of the solvent under reduced pressure and drying in vacuo afforded the title compounds (19:67:14 meta/para/SM) as an orange powder $(121 \mathrm{mg}, 0.20 \mathrm{mmol}$ borylated products, $79 \%, 1: 3.5$ meta/para).

para Product: ${ }^{1} \mathrm{H}$ NMR $\left(600 \mathrm{MHz}, \mathrm{CDCl}_{3}\right) \delta 8.01(\mathrm{~s}, 1 \mathrm{H}), 7.77$ $(\mathrm{d}, J=8.1 \mathrm{~Hz}, 2 \mathrm{H}), 7.69(\mathrm{~d}, J=7.6 \mathrm{~Hz}, 1 \mathrm{H}), 7.52-7.51(\mathrm{~m}, 1 \mathrm{H})$, $7.14(\mathrm{~d}, J=8.0 \mathrm{~Hz}, 2 \mathrm{H}), 4.13(\mathrm{~d}, J=16.5 \mathrm{~Hz}, 2 \mathrm{H}), 2.33(\mathrm{~s}, 3 \mathrm{H}), 2.12$ $(\mathrm{d}, J=14.2 \mathrm{~Hz}, 9 \mathrm{H}), 1.34(\mathrm{~s}, 12 \mathrm{H}) ;{ }^{13} \mathrm{C}\left\{{ }^{1} \mathrm{H}\right\}$ NMR $(101 \mathrm{MHz}$, $\left.\mathrm{CDCl}_{3}\right) \delta 143.7,139.5\left(\mathrm{~d},{ }^{4} J_{\mathrm{C}-\mathrm{P}}=3.1 \mathrm{~Hz}\right), 139.3,134.5\left(\mathrm{~d},{ }^{4} J_{\mathrm{C}-\mathrm{P}}=\right.$ $3.4 \mathrm{~Hz}), 132.0\left(\mathrm{~d},{ }^{3} J_{\mathrm{C}-\mathrm{p}}=4.8 \mathrm{~Hz}\right), 131.9\left(\mathrm{~d},{ }^{2} J_{\mathrm{C}-\mathrm{p}}=9.3 \mathrm{~Hz}\right), 128.7$, $125.8,124.4\left(\mathrm{~d},{ }^{3} J_{\mathrm{C}-\mathrm{P}}=6.4 \mathrm{~Hz}\right), 84.5,30.7\left(\mathrm{~d},{ }^{1} J_{\mathrm{C}-\mathrm{P}}=49.7 \mathrm{~Hz}\right), 24.9$, 21.3, $8.4\left(\mathrm{~d},{ }^{1} J_{\mathrm{C}-\mathrm{P}}=53.7 \mathrm{~Hz}\right) ;{ }^{31} \mathrm{P}$ NMR $\left(243 \mathrm{MHz}, \mathrm{CDCl}_{3}\right) \delta 29.64$.

Trimethyl(2-methyl-5-(4,4,5,5-tetramethyl-1,3,2-dioxaborolan2-yl)benzyl)phosphonium 4-Methylbenzenesulfonate (4d). With 
Sulfonate Ligand 1 with $3 \mathrm{~mol} \%[\operatorname{Ir}(\mathrm{COD}) \mathrm{OMe}]_{2}$ at $70{ }^{\circ} \mathrm{C}$. Following GP2, the compound was formed using trimethyl(2methylbenzyl)phosphonium 4-methylbenzenesulfonate (3d) (88 mg, $0.25 \mathrm{mmol}$ ), $\mathrm{B}_{2} \mathrm{Pin}_{2}$ (127 mg, $0.5 \mathrm{mmol}, 2$ equiv), [ $\left.\mathrm{Ir}(\mathrm{COD}) \mathrm{OMe}\right]_{2}$ (5.0 mg, $0.0075 \mathrm{mmol}, 0.03$ equiv), 1 ( $7.6 \mathrm{mg}, 0.015 \mathrm{mmol}, 0.06$ equiv), and 1,4-dioxane $(1.25 \mathrm{~mL})$. The reaction mixture was stirred for $16 \mathrm{~h}$ at $70^{\circ} \mathrm{C}$. Analysis of crude ${ }^{1} \mathrm{H}$ NMR showed $18: 1$ meta/para borylation. The solvent was removed, and the salts precipitated with $\mathrm{Et}_{2} \mathrm{O}$. This was followed by filtration through a pad of $\mathrm{MgSO}_{4}$ and elution of the salts with $\mathrm{CH}_{2} \mathrm{Cl}_{2}$. Removal of the solvent under reduced pressure and drying in vacuo afforded the title compounds (90:5:5 meta/para/SM) as an orange solid $(111 \mathrm{mg}, 0.22 \mathrm{mmol}$ borylated products, $89 \%, 18: 1$ meta/para).

Compound 4d: ${ }^{1} \mathrm{H}$ NMR $\left(600 \mathrm{MHz}, \mathrm{CDCl}_{3}\right) \delta 7.77(\mathrm{~d}, J=8.2$ $\mathrm{Hz}, 2 \mathrm{H}), 7.64(\mathrm{~d}, J=7.6 \mathrm{~Hz}, 1 \mathrm{H}), 7.49(\mathrm{~d}, J=2.0 \mathrm{~Hz}, 1 \mathrm{H}), 7.22(\mathrm{~d}, J$ $=7.6 \mathrm{~Hz}, 1 \mathrm{H}), 7.13(\mathrm{~d}, J=7.9 \mathrm{~Hz}, 2 \mathrm{H}), 3.81(\mathrm{~d}, J=16.0 \mathrm{~Hz}, 2 \mathrm{H})$, $2.35(\mathrm{~s}, 3 \mathrm{H}), 2.32(\mathrm{~s}, 3 \mathrm{H}), 2.06(\mathrm{~d}, J=14.3 \mathrm{~Hz}, 9 \mathrm{H}), 1.33(\mathrm{~s}, 12 \mathrm{H})$; ${ }^{13} \mathrm{C}\left\{{ }^{1} \mathrm{H}\right\}$ NMR $\left(101 \mathrm{MHz}, \mathrm{CDCl}_{3}\right) \delta 143.9,140.8\left(\mathrm{~d},{ }^{3} J_{\mathrm{C}-\mathrm{P}}=5.5 \mathrm{~Hz}\right)$, $139.2,136.8\left(\mathrm{~d},{ }^{3} J_{\mathrm{C}-\mathrm{P}}=5.0 \mathrm{~Hz}\right), 134.8\left(\mathrm{~d},{ }^{5} J_{\mathrm{C}-\mathrm{P}}=3.7 \mathrm{~Hz}\right), 131.1(\mathrm{~d}$, $\left.{ }^{4} J_{\mathrm{C}-\mathrm{P}}=3.1 \mathrm{~Hz}\right), 128.7,126.6\left(\mathrm{~d},{ }^{2} J_{\mathrm{C}-\mathrm{P}}=8.9 \mathrm{~Hz}\right), 125.9,84.0,27.8(\mathrm{~d}$, $\left.{ }^{1} J_{\mathrm{C}-\mathrm{P}}=49.1 \mathrm{~Hz}\right), 24.9,21.3,20.5,8.1\left(\mathrm{~d},{ }^{1} J_{\mathrm{C}-\mathrm{P}}=54.0 \mathrm{~Hz}\right) ;{ }^{31} \mathrm{P} \mathrm{NMR}$ $\left(243 \mathrm{MHz}, \mathrm{CDCl}_{3}\right) \delta 28.79$; HRMS $m / z$ (ESI+) $[\mathrm{M}-\mathrm{OTs}]^{+}$calcd for $\left[\mathrm{C}_{17} \mathrm{H}_{29} \mathrm{BO}_{2} \mathrm{P}\right]^{+}$307.1993, found 307.1988.

With tmphen with $3 \mathrm{~mol} \%[\operatorname{Ir}(\mathrm{COD}) \mathrm{OMe}]_{2}$ at $70{ }^{\circ} \mathrm{C}$. Following GP2, the compound was formed using trimethyl(2-methylbenzyl)phosphonium 4-methylbenzenesulfonate (3d) $(88 \mathrm{mg}, 0.25 \mathrm{mmol})$, $\mathrm{B}_{2} \mathrm{Pin}_{2}\left(127 \mathrm{mg}, 0.5 \mathrm{mmol}, 2\right.$ equiv), $[\mathrm{Ir}(\mathrm{COD}) \mathrm{OMe}]_{2}(5.0 \mathrm{mg}$, $0.0075 \mathrm{mmol}, 0.03$ equiv), tmphen ( $3.6 \mathrm{mg}, 0.015 \mathrm{mmol}, 0.06$ equiv), and 1,4-dioxane $(1.25 \mathrm{~mL})$. The reaction mixture was stirred for $16 \mathrm{~h}$ at $70{ }^{\circ} \mathrm{C}$. Analysis of crude ${ }^{1} \mathrm{H}$ NMR showed 1:1.4 meta/para borylation. The solvent was removed, and the salts precipitated with $\mathrm{Et}_{2} \mathrm{O}$. This was followed by filtration through a pad of $\mathrm{MgSO}_{4}$ and elution of the salts with $\mathrm{CH}_{2} \mathrm{Cl}_{2}$. Removal of the solvent under reduced pressure and drying in vacuo afforded the title compounds (30:42:28 meta/para/SM) as a brown solid $(104 \mathrm{mg}, 0.17 \mathrm{mmol}$ borylated products, $68 \%, 1: 1.3$ meta $/$ para $)$.

para Product: ${ }^{1} \mathrm{H}$ NMR $\left(600 \mathrm{MHz}, \mathrm{CDCl}_{3}\right) \delta 7.76(\mathrm{~d}, J=8.1 \mathrm{~Hz}$, $2 \mathrm{H}), 7.63(\mathrm{~s}, 1 \mathrm{H}), 7.55(\mathrm{~d}, J=7.6 \mathrm{~Hz}, 1 \mathrm{H}), 7.19-7.17(\mathrm{~m}, 1 \mathrm{H}), 7.13$ $(\mathrm{d}, J=7.9 \mathrm{~Hz}, 2 \mathrm{H}), 3.95(\mathrm{~d}, J=16.7 \mathrm{~Hz}, 2 \mathrm{H}), 2.32(\mathrm{~s}, 3 \mathrm{H}), 2.30(\mathrm{~s}$, $3 \mathrm{H}), 2.03(\mathrm{~d}, J=14.3 \mathrm{~Hz}, 9 \mathrm{H}), 1.34(\mathrm{~s}, 12 \mathrm{H}) ;{ }^{13} \mathrm{C}\left\{{ }^{1} \mathrm{H}\right\}$ NMR $(101$ $\left.\mathrm{MHz}, \mathrm{CDCl}_{3}\right) \delta 143.9,139.3,137.7\left(\mathrm{~d},{ }^{4} J_{\mathrm{C}-\mathrm{P}}=3.3 \mathrm{~Hz}\right), 136.4(\mathrm{~d}$, $\left.{ }^{3} J_{\mathrm{C}-\mathrm{P}}=5.7 \mathrm{~Hz}\right), 132.8\left(\mathrm{~d},{ }^{4} J_{\mathrm{C}-\mathrm{P}}=3.4 \mathrm{~Hz}\right), 130.5\left(\mathrm{~d},{ }^{3} J_{\mathrm{C}-\mathrm{P}}=4.7 \mathrm{~Hz}\right)$, $130.4\left(\mathrm{~d},{ }^{2} J_{\mathrm{C}-\mathrm{P}}=9.1 \mathrm{~Hz}\right), 128.7,125.8,84.0,27.7\left(\mathrm{~d},{ }^{1} J_{\mathrm{C}-\mathrm{P}}=49.0\right.$ $\mathrm{Hz}), 24.9,21.3,19.9,8.0\left(\mathrm{~d},{ }^{1} J_{\mathrm{C}-\mathrm{P}}=53.9 \mathrm{~Hz}\right) ;{ }^{31} \mathrm{P}$ NMR $(243 \mathrm{MHz}$, $\left.\mathrm{CDCl}_{3}\right) \delta 29.12$.

(3-Fluoro-5-(4,4,5,5-tetramethyl-1,3,2-dioxaborolan-2-yl)benzyl)trimethylphosphonium 4-Methylbenzenesulfonate (4e). With Sulfonate Ligand 1. Following GP2, the compound was formed using (3-fluorobenzyl)trimethylphosphonium 4-methylbenzenesulfonate (3e) $(89 \mathrm{mg}, 0.25 \mathrm{mmol}), \mathrm{B}_{2} \mathrm{Pin}_{2}(95 \mathrm{mg}, 0.375 \mathrm{mmol}$, 1.5 equiv $),[\operatorname{Ir}(\mathrm{COD}) \mathrm{OMe}]_{2}(2.5 \mathrm{mg}, 0.00375 \mathrm{mmol}, 0.015$ equiv $), 1$ (3.8 $\mathrm{mg}, 0.0075 \mathrm{mmol}, 0.03$ equiv), and 1,4-dioxane $(1.25 \mathrm{~mL})$. The reaction mixture was stirred for $16 \mathrm{~h}$ at $50{ }^{\circ} \mathrm{C}$. Analysis of crude ${ }^{1} \mathrm{H}$ NMR showed $>20: 1$ meta/para borylation. The solvent was removed, and the salts precipitated with $\mathrm{Et}_{2} \mathrm{O}$. This was followed by filtration through a pad of $\mathrm{MgSO}_{4}$ and elution of the salts with $\mathrm{CHCl}_{3}$. Removal of the solvent under reduced pressure and drying in vacuo afforded the meta product and starting material (84:16 meta/SM, para isomer was not detected) as a light orange solid $(93 \mathrm{mg}, 0.17 \mathrm{mmol}$ borylated products, $68 \%,>20: 1$ meta/para).

Compound 4e: ${ }^{1} \mathrm{H}$ NMR $\left(600 \mathrm{MHz}, \mathrm{CDCl}_{3}\right) \delta 7.80(\mathrm{~d}, J=8.1 \mathrm{~Hz}$, $2 \mathrm{H}), 7.44-7.42(\mathrm{~m}, 1 \mathrm{H}), 7.35(\mathrm{~s}, 1 \mathrm{H}), 7.22-7.20(\mathrm{~m}, 1 \mathrm{H}), 7.17(\mathrm{~d}, J$ $=8.1 \mathrm{~Hz}, 2 \mathrm{H}), 3.94(\mathrm{~d}, J=16.3 \mathrm{~Hz}, 2 \mathrm{H}), 2.33(\mathrm{~s}, 3 \mathrm{H}), 2.03(\mathrm{~d}, J=$ $14.3 \mathrm{~Hz}, 9 \mathrm{H}), 1.34(\mathrm{~s}, 12 \mathrm{H}) ;{ }^{13} \mathrm{C}\left\{{ }^{1} \mathrm{H}\right\} \operatorname{NMR}\left(101 \mathrm{MHz}, \mathrm{CDCl}_{3}\right) \delta$ $162.7\left(\mathrm{dd},{ }^{1} J_{\mathrm{C}-\mathrm{F}}=249.7 \mathrm{~Hz},{ }^{4} J_{\mathrm{C}-\mathrm{P}}=3.7 \mathrm{~Hz}\right), 143.7,139.5,131.3(\mathrm{dd}$, $\left.{ }^{4} J_{\mathrm{C}-\mathrm{F}}=3.0 \mathrm{~Hz},{ }^{3} J_{\mathrm{C}-\mathrm{P}}=5.3 \mathrm{~Hz}\right), 130.6\left(\mathrm{dd},{ }^{3} J_{\mathrm{C}-\mathrm{F}}=7.6 \mathrm{~Hz},{ }^{2} J_{\mathrm{C}-\mathrm{P}}=9.1\right.$ $\mathrm{Hz}), 128.8,125.8,121.2\left(\mathrm{dd},{ }^{2} J_{\mathrm{C}-\mathrm{F}}=18.5 \mathrm{~Hz},{ }^{5} \mathrm{~J}_{\mathrm{C}-\mathrm{P}}=3.1 \mathrm{~Hz}\right), 120.2$ $\left(\mathrm{dd},{ }^{2} J_{\mathrm{C}-\mathrm{F}}=23.0 \mathrm{~Hz},{ }^{3} J_{\mathrm{C}-\mathrm{P}}=5.4 \mathrm{~Hz}\right), 84.4,30.0\left(\mathrm{~d},{ }^{1} J_{\mathrm{C}-\mathrm{P}}=48.5 \mathrm{~Hz}\right)$, 24.9, 21.3, $7.8\left(\mathrm{~d},{ }^{1} J_{\mathrm{C}-\mathrm{P}}=54.5 \mathrm{~Hz}\right) ;{ }^{19} \mathrm{~F} \mathrm{NMR}\left(376 \mathrm{MHz}, \mathrm{CDCl}_{3}\right) \delta$
-112.56; ${ }^{31} \mathrm{P}$ NMR $\left(243 \mathrm{MHz}, \mathrm{CDCl}_{3}\right) \delta 27.53$; HRMS $m / z$ (ESI+) $[\mathrm{M}-\mathrm{OTs}]^{+}$calcd for $\left[\mathrm{C}_{16} \mathrm{H}_{26} \mathrm{BFO}_{2} \mathrm{P}\right]^{+} 311.1742$, found 311.1736 .

With tmphen. Following GP2, the compound was formed using (3-fluorobenzyl)trimethylphosphonium 4-methylbenzenesulfonate (3e) $(89 \mathrm{mg}, 0.25 \mathrm{mmol}), \mathrm{B}_{2} \operatorname{Pin}_{2}$ (95 mg, $0.375 \mathrm{mmol}, 1.5$ equiv), $[\mathrm{Ir}(\mathrm{COD}) \mathrm{OMe}]_{2}(2.5 \mathrm{mg}, 0.00375 \mathrm{mmol}, 0.015$ equiv $)$, tmphen $(1.8$ $\mathrm{mg}, 0.0075 \mathrm{mmol}, 0.03$ equiv), and 1,4-dioxane $(1.25 \mathrm{~mL})$. The reaction mixture was stirred for $16 \mathrm{~h}$ at $50{ }^{\circ} \mathrm{C}$. Analysis of crude ${ }^{1} \mathrm{H}$ NMR showed a ratio 1:1.3:2.6 of meta/para/starting material. The solvent was removed, and the salts precipitated with $\mathrm{Et}_{2} \mathrm{O}$. This was followed by filtration through a pad of $\mathrm{MgSO}_{4}$ and elution of the salts with $\mathrm{CHCl}_{3}$. Removal of the solvent under reduced pressure and drying in vacuo afforded the title compounds (20:26:54 meta/para) $\mathrm{SM})$ as a brown solid ( $84 \mathrm{mg}, 0.09 \mathrm{mmol}$ borylated products, $37 \%$, 1:1.3 meta/para).

para Product: ${ }^{1} \mathrm{H}$ NMR $\left(600 \mathrm{MHz}, \mathrm{CDCl}_{3}\right) \delta 7.77(\mathrm{~d}, J=8.0 \mathrm{~Hz}$, $2 \mathrm{H}), 7.64(\mathrm{t}, J=6.4 \mathrm{~Hz}, 1 \mathrm{H}), 7.15(\mathrm{~d}, J=8.0 \mathrm{~Hz}, 2 \mathrm{H}), 7.03(\mathrm{~d}, J=7.0$ $\mathrm{Hz}, 1 \mathrm{H}), 6.93(\mathrm{~d}, J=9.1 \mathrm{~Hz}, 1 \mathrm{H}), 4.02(\mathrm{~d}, J=16.9 \mathrm{~Hz}, 2 \mathrm{H}), 2.33(\mathrm{~s}$, $3 \mathrm{H}), 1.97(\mathrm{~d}, J=14.4 \mathrm{~Hz}, 9 \mathrm{H}), 1.35(\mathrm{~s}, 12 \mathrm{H}) ;{ }^{13} \mathrm{C}\left\{{ }^{1} \mathrm{H}\right\}$ NMR $(101$ $\left.\mathrm{MHz}, \mathrm{CDCl}_{3}\right) \delta 167.2\left(\mathrm{dd},{ }^{1} J_{\mathrm{C}-\mathrm{F}}=253.0 \mathrm{~Hz},{ }^{4} J_{\mathrm{C}-\mathrm{P}}=4.0 \mathrm{~Hz}\right), 143.7$, $139.6,137.7\left(\mathrm{dd},{ }^{3} J_{\mathrm{C}-\mathrm{F}}=8.6 \mathrm{~Hz},{ }^{4} J_{\mathrm{C}-\mathrm{P}}=3.7 \mathrm{~Hz}\right), 134.7\left(\mathrm{dd},{ }^{3} J_{\mathrm{C}-\mathrm{F}}=\right.$ $\left.9.2 \mathrm{~Hz},{ }^{2} J_{\mathrm{C}-\mathrm{P}}=9.2 \mathrm{~Hz}\right), 128.9,125.8,125.6\left(\mathrm{dd},{ }^{4} J_{\mathrm{C}-\mathrm{F}}=4.4 \mathrm{~Hz},{ }^{3} J_{\mathrm{C}-\mathrm{P}}\right.$ $=4.4 \mathrm{~Hz}), 116.9\left(\mathrm{dd},{ }^{2} J_{\mathrm{C}-\mathrm{F}}=23.6 \mathrm{~Hz},{ }^{3} J_{\mathrm{C}-\mathrm{P}}=5.5 \mathrm{~Hz}\right), 84.1,29.6(\mathrm{~d}$, $\left.{ }^{1} J_{\mathrm{C}-\mathrm{P}}=49.2 \mathrm{~Hz}\right), 24.8,21.3,7.5\left(\mathrm{~d},{ }^{1} J_{\mathrm{C}-\mathrm{P}}=54.4 \mathrm{~Hz}\right) ;{ }^{19} \mathrm{~F}$ NMR $(376$ $\left.\mathrm{MHz}, \mathrm{CDCl}_{3}\right) \delta-101.74\left(\mathrm{~d},{ }^{5} J_{\mathrm{F}-\mathrm{P}}=2.9 \mathrm{~Hz}\right) ;{ }^{31} \mathrm{P} \mathrm{NMR}(243 \mathrm{MHz}$, $\left.\mathrm{CDCl}_{3}\right) \delta 27.84$.

(3,5-Bis(4,4,5,5-tetramethyl-1,3,2-dioxaborolan-2-yl)benzyl)trimethylphosphonium 4-Methylbenzenesulfonate (4f). With Sulfonate Ligand L1. Following GP2, the compound was formed using benzyltrimethylphosphonium 4-methylbenzenesulfonate (3f) $(85 \mathrm{mg}$, $0.25 \mathrm{mmol}$ ), $\mathrm{B}_{2} \mathrm{Pin}_{2}(190 \mathrm{mg}, 0.75 \mathrm{mmol}, 3.0$ equiv), [ $\operatorname{Ir}(\mathrm{COD})$ $\mathrm{OMe}_{2}(2.5 \mathrm{mg}, 0.00375 \mathrm{mmol}, 0.015$ equiv), 1 (3.8 $\mathrm{mg}, 0.0075$ mmol, 0.03 equiv), and 1,4-dioxane $(1.25 \mathrm{~mL})$. The reaction mixture was stirred for $16 \mathrm{~h}$ at $50{ }^{\circ} \mathrm{C}$. Analysis of crude ${ }^{1} \mathrm{H}$ NMR showed $>20: 1$ dimeta/para borylation. The solvent was removed, and the salts precipitated with $\mathrm{Et}_{2} \mathrm{O}$. This was followed by filtration through a pad of $\mathrm{MgSO}_{4}$ and elution of the salts with $\mathrm{CHCl}_{3}$. Removal of the solvent under reduced pressure and drying in vacuo afforded the title compound (contaminated with $<10 \%$ mono meta product) as a light orange solid $(111 \mathrm{mg}, 0.17 \mathrm{mmol}$ diborylated product, $69 \%,>20: 1$ dimeta + monometa/para)

Compound 4f: ${ }^{1} \mathrm{H}$ NMR (600 MHz, $\left.\mathrm{CDCl}_{3}\right) \delta 8.22(\mathrm{~s}, 1 \mathrm{H}), 7.81$ $(\mathrm{d}, J=8.1 \mathrm{~Hz}, 2 \mathrm{H}), 7.69(\mathrm{~d}, J=1.9 \mathrm{~Hz}, 2 \mathrm{H}), 7.15(\mathrm{~d}, J=8.0 \mathrm{~Hz}, 2 \mathrm{H})$ $3.73(\mathrm{~d}, J=15.7 \mathrm{~Hz}, 2 \mathrm{H}), 2.31(\mathrm{~s}, 3 \mathrm{H}), 2.04(\mathrm{~d}, J=14.3 \mathrm{~Hz}, 9 \mathrm{H})$, $1.33(\mathrm{~s}, 24 \mathrm{H}) ;{ }^{13} \mathrm{C}\left\{{ }^{1} \mathrm{H}\right\}$ NMR $\left(151 \mathrm{MHz}, \mathrm{CDCl}_{3}\right) \delta 143.7,141.2(\mathrm{~d}$, $\left.{ }^{5} J_{\mathrm{C}-\mathrm{P}}=3.5 \mathrm{~Hz}\right), 139.2,138.6\left(\mathrm{~d},{ }^{3} J_{\mathrm{C}-\mathrm{P}}=5.2 \mathrm{~Hz}\right), 128.7,127.2(\mathrm{~d}$, $\left.{ }^{2} \mathrm{~J}_{\mathrm{C}-\mathrm{P}}=8.9 \mathrm{~Hz}\right), 125.9,84.1,30.8\left(\mathrm{~d},{ }^{1} \mathrm{~J}_{\mathrm{C}-\mathrm{P}}=49.2 \mathrm{~Hz}\right), 24.9,21.3,7.8$ $\left(\mathrm{d},{ }^{1} J_{\mathrm{C}-\mathrm{P}}=54.6 \mathrm{~Hz}\right) ;{ }^{31} \mathrm{P}$ NMR $\left(243 \mathrm{MHz}, \mathrm{CDCl}_{3}\right)$ 26.98; HRMS $\mathrm{m} /$ $z$ (ESI+) $[\mathrm{M}-\mathrm{OTs}]^{+}$calcd for $\left[\mathrm{C}_{22} \mathrm{H}_{38} \mathrm{~B}_{2} \mathrm{O}_{4} \mathrm{P}\right]^{+} 419.2688$, found 419.2685

With tmphen. Following GP2, the compound was formed using benzyltrimethylphosphonium 4-methylbenzenesulfonate (3f) $(85 \mathrm{mg}$, $0.25 \mathrm{mmol}$ ), $\mathrm{B}_{2} \operatorname{Pin}_{2}$ (190 mg, $0.75 \mathrm{mmol}, 3.0$ equiv), [ $\operatorname{Ir}(\mathrm{COD})-$ $\mathrm{OMe}_{2}$ (2.5 mg, $0.00375 \mathrm{mmol}, 0.015$ equiv), tmphen (1.8 mg, $0.0075 \mathrm{mmol}, 0.03$ equiv), and 1,4-dioxane $(1.25 \mathrm{~mL})$. The reaction mixture was stirred for $16 \mathrm{~h}$ at $50{ }^{\circ} \mathrm{C}$. The solvent was removed, and the salts precipitated with $\mathrm{Et}_{2} \mathrm{O}$. This was followed by filtration through a pad of $\mathrm{MgSO}_{4}$ and elution of the salts with $\mathrm{CHCl}_{3}$. Removal of the solvent under reduced pressure and drying in vacuo afforded a dark brown solid. NMR analysis revealed that this was a complex mixture of products (presumably a mixture of the starting material and meta, dimeta, and para products).

Trimethyl((4-(4,4,5,5-tetramethyl-1,3,2-dioxaborolan-2-yl)pyridin-2-yl)methyl)phosphonium 4-Methylbenzenesulfonate (6a). With Sulfonate Ligand 1. Following GP2, the compound was formed using trimethyl(pyridin-2-ylmethyl)phosphonium 4-methylbenzenesulfonate (5a) (85 mg, $0.25 \mathrm{mmol}$ ), $\mathrm{B}_{2} \mathrm{Pin}_{2}(95 \mathrm{mg}, 0.375 \mathrm{mmol}, 1.5$ equiv), $[\mathrm{Ir}(\mathrm{COD}) \mathrm{OMe}]_{2}(2.5 \mathrm{mg}, 0.00375 \mathrm{mmol}, 0.015$ equiv $), 1$ (3.8 $\mathrm{mg}, 0.0075 \mathrm{mmol}, 0.03$ equiv), and 1,4-dioxane $(1.25 \mathrm{~mL})$. The reaction mixture was stirred for $1 \mathrm{~h}$ at $50{ }^{\circ} \mathrm{C}$. Analysis of the crude ${ }^{1} \mathrm{H}$ 
NMR with 1,2-dimethoxyethane as the internal standard showed 10:1 C4/C5 borylation, in 53\% NMR yield (NMR yield used in this case due to possible contamination by the dimeta product). For isolation of the product, the solvent was removed. The resultant brown oil was washed with $\mathrm{Et}_{2} \mathrm{O}$ (add $\mathrm{Et}_{2} \mathrm{O}$, decant off $\mathrm{Et}_{2} \mathrm{O}$, repeat 10-15 times to remove most of the residual $\mathrm{B}_{2} \mathrm{Pin}_{2}$ ). Drying in vacuo afforded the title compounds as an orange powder initially, which became a brown oil upon standing.

Compound 6a: ${ }^{1} \mathrm{H}$ NMR $\left(400 \mathrm{MHz}, \mathrm{CDCl}_{3}\right) \delta 8.49$ (dd, $J=4.8$, $1.0 \mathrm{~Hz}, 1 \mathrm{H}), 7.77(\mathrm{~d}, J=8.1 \mathrm{~Hz}, 2 \mathrm{H}), 7.65(\mathrm{~s}, 1 \mathrm{H}), 7.56-7.55(\mathrm{~m}$, $1 \mathrm{H}), 7.12(\mathrm{~d}, J=7.9 \mathrm{~Hz}, 2 \mathrm{H}), 4.01(\mathrm{~d}, J=15.4 \mathrm{~Hz}, 2 \mathrm{H}), 2.31(\mathrm{~s}, 3 \mathrm{H})$, $2.14(\mathrm{~d}, J=14.6 \mathrm{~Hz}, 9 \mathrm{H}), 1.34(\mathrm{~s}, 12 \mathrm{H}) ;{ }^{13} \mathrm{C}\left\{{ }^{1} \mathrm{H}\right\} \mathrm{NMR}(101 \mathrm{MHz}$, $\left.\mathrm{CDCl}_{3}\right) \delta 150.3\left(\mathrm{~d},{ }^{2} J_{\mathrm{C}-\mathrm{P}}=8.9 \mathrm{~Hz}\right), 148.8\left(\mathrm{~d},{ }^{4} J_{\mathrm{C}-\mathrm{P}}=1.1 \mathrm{~Hz}\right), 143.7$, $139.2,129.9\left(\mathrm{~d},{ }^{3} J_{\mathrm{C}-\mathrm{P}}=7.6 \mathrm{~Hz}\right), 128.7,127.9\left(\mathrm{~d},{ }^{5} J_{\mathrm{C}-\mathrm{P}}=1.7 \mathrm{~Hz}\right)$, $125.9,84.8,32.4\left(\mathrm{~d},{ }^{1} J_{\mathrm{C}-\mathrm{P}}=54.2 \mathrm{~Hz}\right), 24.9,21.3,9.2\left(\mathrm{~d},{ }^{1} J_{\mathrm{C}-\mathrm{P}}=55.1\right.$ $\mathrm{Hz}) ;{ }^{31} \mathrm{P}$ NMR $\left(162 \mathrm{MHz}, \mathrm{CDCl}_{3}\right) \delta 27.36$; HRMS $m / z$ (ESI+) [M - OTs $]^{+}$calcd for $\left[\mathrm{C}_{15} \mathrm{H}_{26} \mathrm{BNO}_{2} \mathrm{P}\right]^{+}$294.1789, found 294.1777.

Note that the peaks partially overlap with the SM in the ${ }^{1} \mathrm{H}$ NMR spectrum

With tmphen. Following GP2, the compound was formed using trimethyl(pyridin-2-ylmethyl)phosphonium 4-methylbenzenesulfonate (5a) ( $85 \mathrm{mg}, 0.25 \mathrm{mmol}), \mathrm{B}_{2} \operatorname{Pin}_{2}(95 \mathrm{mg}, 0.375 \mathrm{mmol}, 1.5$ equiv), $[\mathrm{Ir}(\mathrm{COD}) \mathrm{OMe}]_{2}(2.5 \mathrm{mg}, 0.00375 \mathrm{mmol}, 0.015$ equiv $)$, tmphen $(1.8 \mathrm{mg}, 0.0075 \mathrm{mmol}, 0.03$ equiv). and 1,4-dioxane (1.25 $\mathrm{mL}$ ). The reaction mixture was stirred for $1 \mathrm{~h}$ at $50{ }^{\circ} \mathrm{C}$. Analysis of the crude ${ }^{1} \mathrm{H}$ NMR with 1,2-dimethoxyethane as the internal standard showed 1:1.3 C4/C5 borylation, in 60\% NMR yield. For isolation of the product, the solvent was removed. The resultant brown oil was washed with $\mathrm{Et}_{2} \mathrm{O}$. Drying in vacuo afforded the title compounds as an orange powder initially. This powder became a brown oil upon standing.

C5 Product: ${ }^{1} \mathrm{H}$ NMR $\left(400 \mathrm{MHz}, \mathrm{CDCl}_{3}\right) \delta 8.77(\mathrm{~s}, 1 \mathrm{H}), 7.96$ (d, $J=7.7 \mathrm{~Hz}, 1 \mathrm{H}), 7.72(\mathrm{~d}, J=8.1 \mathrm{~Hz}, 2 \mathrm{H}), 7.38(\mathrm{~d}, J=7.7 \mathrm{~Hz}, 1 \mathrm{H})$, $7.18(\mathrm{~d}, J=8.0 \mathrm{~Hz}, 2 \mathrm{H}), 4.07(\mathrm{~d}, J=16.0 \mathrm{~Hz}, 2 \mathrm{H}), 2.29(\mathrm{~s}, 3 \mathrm{H}), 2.05$ $(\mathrm{d}, J=14.6 \mathrm{~Hz}, 9 \mathrm{H}), 1.33(\mathrm{~s}, 12 \mathrm{H}) ;{ }^{13} \mathrm{C}\left\{{ }^{1} \mathrm{H}\right\}$ NMR $(101 \mathrm{MHz}$, $\left.\mathrm{CDCl}_{3}\right) \delta 155.1\left(\mathrm{~d},{ }^{4} J_{\mathrm{C}-\mathrm{P}}=1.7 \mathrm{~Hz}\right), 153.5\left(\mathrm{~d},{ }^{2} J_{\mathrm{C}-\mathrm{P}}=9.2 \mathrm{~Hz}\right), 143.7$, 143.6, 139.4, 128.7, 125.8, $124.7\left(\mathrm{~d},{ }^{3} J_{\mathrm{C}-\mathrm{P}}=7.1 \mathrm{~Hz}\right), 84.4,32.4(\mathrm{~d}$, $\left.{ }^{4} J_{\mathrm{C}-\mathrm{P}}=52.8 \mathrm{~Hz}\right), 24.9,21.3,8.8\left(\mathrm{~d},{ }^{4} J_{\mathrm{C}-\mathrm{P}}=54.9 \mathrm{~Hz}\right) ;{ }^{31} \mathrm{P} \mathrm{NMR}(162$ $\left.\mathrm{MHz}, \mathrm{CDCl}_{3}\right) \delta 27.58$.

Trimethyl((5-(4,4,5,5-tetramethyl-1,3,2-dioxaborolan-2-yl)-2trifluoromethyl)phenethyl)phosphonium 4-Methylbenzenesulfonate (8a). With Sulfonate Ligand $1(0.25 \mathrm{mmol}$ Scale). Following $\mathrm{GP2}$, the compound was formed using $7 \mathrm{a}(105.1 \mathrm{mg}, 0.25 \mathrm{mmol})$, $\mathrm{B}_{2} \mathrm{Pin}_{2}(127.0 \mathrm{mg}, 0.50 \mathrm{mmol}),[\mathrm{Ir}(\mathrm{COD}) \mathrm{OMe}]_{2}(2.5 \mathrm{mg}, 0.00375$ $\mathrm{mmol})$, and 1 (3.8 $\mathrm{mg}, 0.0075 \mathrm{mmol})$ in dioxane $(1.25 \mathrm{~mL})$. The mixture was stirred in a vial at $70{ }^{\circ} \mathrm{C}$ for $20 \mathrm{~h}$. Analysis of crude ${ }^{1} \mathrm{H}$ NMR showed a 26:1:2 ratio of meta/para/starting material (26:1 meta/para borylation). Purification by evaporation of solvent and addition of ether, followed by filtration and drying in vacuo, gave the title compound as a light brown solid (109.2 mg, $0.200 \mathrm{mmol}, 80 \%)$, as a mixture of a 42:1:2.3 ratio of meta/para/starting material.

Compound 8a: ${ }^{1} \mathrm{H}$ NMR $\left(600 \mathrm{MHz}, \mathrm{CD}_{3} \mathrm{OD}\right) \delta 7.91(\mathrm{~s}, 1 \mathrm{H})$, $7.81(\mathrm{~d}, J=7.9 \mathrm{~Hz}, 1 \mathrm{H}), 7.71(\mathrm{~d}, J=8.0 \mathrm{~Hz}, 1 \mathrm{H}), 7.69(\mathrm{~d}, J=8.1 \mathrm{~Hz}$, $2 \mathrm{H}), 7.19(\mathrm{~d}, J=8.1 \mathrm{~Hz}, 2 \mathrm{H}), 3.06-3.08(\mathrm{~m}, 2 \mathrm{H}), 2.48-2.51(\mathrm{~m}$, $2 \mathrm{H}), 2.34(\mathrm{~s}, 3 \mathrm{H}), 1.95\left(\mathrm{~d},{ }^{2} \mathrm{~J}_{\mathrm{PH}}=14.6 \mathrm{~Hz}, 9 \mathrm{H}\right), 1.36(\mathrm{~s}, 12 \mathrm{H})$; ${ }^{13} \mathrm{C}\left\{{ }^{1} \mathrm{H}\right\}$ NMR $\left(151 \mathrm{MHz}, \mathrm{CD}_{3} \mathrm{OD}\right) \delta 142.4,140.1,137.10,137.12$ $(\mathrm{d}, J=17.0 \mathrm{~Hz}), 133.2,130.0\left(\mathrm{q},{ }^{2} J_{\mathrm{C}-\mathrm{F}}=29.9 \mathrm{~Hz}\right), 128.4,125.5$, $125.2\left(\mathrm{q},{ }^{3} \mathrm{~J}_{\mathrm{C}-\mathrm{F}}=5.6 \mathrm{~Hz}\right), 124.5\left(\mathrm{q},{ }^{1} J_{\mathrm{C}-\mathrm{F}}=273 \mathrm{~Hz}\right), 84.3,24.8(\mathrm{~d}$, $\left.{ }^{1} J_{\mathrm{C}-\mathrm{P}}=50.8 \mathrm{~Hz}\right), 23.8,23.7,19.9,6.2\left(\mathrm{~d},{ }^{1} J_{\mathrm{C}-\mathrm{P}}=54.7 \mathrm{~Hz}\right) ;{ }^{31} \mathrm{P} \mathrm{NMR}$ $\left(243 \mathrm{MHz}, \mathrm{CD}_{3} \mathrm{OD}\right) \delta 27.31$; HRMS (ESI+) calcd for $[\mathrm{M}-\mathrm{OTs}]^{+}$ $\left[\mathrm{C}_{18} \mathrm{H}_{28} \mathrm{BF}_{3} \mathrm{O}_{2} \mathrm{P}\right]^{+}$375.1872, found 375.1879.

With tmphen Ligand $(0.25 \mathrm{mmol}$ Scale). Following GP2, the compound was formed using $7 \mathrm{a}(105.1 \mathrm{mg}, 0.25 \mathrm{mmol}), \mathrm{B}_{2} \mathrm{Pin}_{2}$ $(127.0 \mathrm{mg}, 0.50 \mathrm{mmol}),[\mathrm{Ir}(\mathrm{COD}) \mathrm{OMe}]_{2}(2.5 \mathrm{mg}, 0.00375 \mathrm{mmol})$, and tmphen $(2.0 \mathrm{mg}, 0.0075 \mathrm{mmol})$ in dioxane $(1.25 \mathrm{~mL})$. The mixture was stirred in a vial at $70{ }^{\circ} \mathrm{C}$ for $20 \mathrm{~h}$. Analysis of crude ${ }^{1} \mathrm{H}$ NMR showed a 1:2:1 ratio of meta/para/starting material. A small sample was triturated with diethyl ether in order to characterize the para isomer. This contained a 4:1:3 ratio of para/meta/starting material. para Product: ${ }^{1} \mathrm{H}$ NMR $\left(600 \mathrm{MHz}, \mathrm{CD}_{3} \mathrm{OD}\right) \delta 8.02(\mathrm{~s}, 1 \mathrm{H}), 7.92$ $(\mathrm{d}, J=7.9 \mathrm{~Hz}, 1 \mathrm{H}), 7.67-7.70(\mathrm{~m}, 3 \mathrm{H}), 7.18(\mathrm{~d}, J=8.0 \mathrm{~Hz}, 2 \mathrm{H})$, $3.04-3.10(\mathrm{~m}, 2 \mathrm{H}), 2.44-2.54(\mathrm{~m}, 2 \mathrm{H}), 2.33(\mathrm{~s}, 3 \mathrm{H}), 1.93(\mathrm{~d}, J=$ $15.0 \mathrm{~Hz}, 9 \mathrm{H}), 1.36(\mathrm{~s}, 12 \mathrm{H}) ;{ }^{13} \mathrm{C}\left\{{ }^{1} \mathrm{H}\right\}$ NMR $\left(151 \mathrm{MHz}, \mathrm{CD}_{3} \mathrm{OD}\right) \delta$ $142.4,141.0(\mathrm{~d}, J=17.2 \mathrm{~Hz}), 140.2,138.5,131.6\left(\mathrm{q},{ }^{3} J_{\mathrm{C}-\mathrm{F}}=5.5 \mathrm{~Hz}\right)$, $130.7,128.4,127.2(\mathrm{q}, J=29.5 \mathrm{~Hz}), 124.5\left(\mathrm{q},{ }^{1} J_{\mathrm{C}-\mathrm{F}}=273 \mathrm{~Hz}\right) 125.5$, $84.3,24.4\left(\mathrm{~d},{ }^{1} \mathrm{~J}_{\mathrm{C}-\mathrm{P}}=50.4 \mathrm{~Hz}\right), 23.8,23.7,19.9,6.2\left(\mathrm{~d},{ }^{1} J_{\mathrm{C}-\mathrm{P}}=54.6\right.$ $\mathrm{Hz})$.

Trimethyl(phenyl)phosphonium 4-Methylbenzenesulfonate (7b). Dimethylphenylphosphine $(0.71 \mathrm{~mL}, 5 \mathrm{mmol})$ was dissolved in methanol $(10 \mathrm{~mL})$; then methyl $p$-toluenesulfonate $(1.37 \mathrm{~mL}, 6$ $\mathrm{mmol}$ ) was added, and the resulting reaction mixture was stirred at room temperature for $16 \mathrm{~h}$. The crude was concentrated (to about 1 $\mathrm{mL})$; then diethyl ether $(20 \mathrm{~mL})$ was added, and the resulting precipitate was collected by filtration and washed with more diethyl ether to afford the title product as a white solid $(1.58 \mathrm{~g}, 4.87 \mathrm{mmol}$, 97\%): ${ }^{1} \mathrm{H}$ NMR (400 MHz, $\left.\mathrm{CDCl}_{3}\right) \delta 7.84(\mathrm{dd}, J=13.1,7.3 \mathrm{~Hz}, 2 \mathrm{H})$, $7.68(\mathrm{~d}, J=8.0 \mathrm{~Hz}, 2 \mathrm{H}), 7.58(\mathrm{dd}, J=7.3,1.2 \mathrm{~Hz}, 1 \mathrm{H}), 7.45(\mathrm{dd}, J=$ 7.9, $3.0 \mathrm{~Hz}, 2 \mathrm{H}), 7.07(\mathrm{~d}, J=8.0 \mathrm{~Hz}, 2 \mathrm{H}), 2.36(\mathrm{~d}, J=14.6 \mathrm{~Hz}, 9 \mathrm{H})$, $2.30(\mathrm{~s}, 3 \mathrm{H}) ;{ }^{13} \mathrm{C}\left\{{ }^{1} \mathrm{H}\right\}$ NMR $\left(101 \mathrm{MHz}, \mathrm{CDCl}_{3}\right) \delta 144.0,139.1$, $133.9\left(\mathrm{~d},{ }^{4} J_{\mathrm{C}-\mathrm{P}}=3.0 \mathrm{~Hz}\right), 131.0\left(\mathrm{~d},{ }^{2} J_{\mathrm{C}-\mathrm{P}}=10.4 \mathrm{~Hz}\right), 129.8\left(\mathrm{~d},{ }^{3} J_{\mathrm{C}-\mathrm{P}}\right.$ $=12.5 \mathrm{~Hz}), 128.6,125.9,122.3\left(\mathrm{~d},{ }^{1} J_{\mathrm{C}-\mathrm{P}}=85.5 \mathrm{~Hz}\right), 21.3,9.6(\mathrm{~d}$, $\left.{ }^{1} J_{\mathrm{C}-\mathrm{P}}=55.8 \mathrm{~Hz}\right) ;{ }^{31} \mathrm{P}\left(162 \mathrm{MHz}, \mathrm{CDCl}_{3}\right) \delta 22.47$; HRMS (ESI+) calcd for $[\mathrm{M}-\mathrm{OTs}]^{+}\left[\mathrm{C}_{9} \mathrm{H}_{14} \mathrm{P}\right]^{+}$153.0828, found 153.0827.

3,5-Bis ((4,4,5,5-tetramethyl-1,3,2-dioxaborolan-2-yl)phenyl)trimethylphosphonium 4-Methylbenzenesulfonate (8b). Following GP2, the compound was formed using $7 \mathbf{b}(81 \mathrm{mg}, 0.25 \mathrm{mmol})$, $\mathrm{B}_{2} \mathrm{Pin}_{2}$ (190 mg, $\left.\left.0.75 \mathrm{mmol}\right), \operatorname{Ir}(\mathrm{COD}) \mathrm{OMe}\right]_{2}(2.5 \mathrm{mg}, 0.00375$ $\mathrm{mmol})$, and $1(3.8 \mathrm{mg}, 0.0075 \mathrm{mmol})$ in dioxane $(1.25 \mathrm{~mL})$. The mixture was stirred in a vial at $50{ }^{\circ} \mathrm{C}$ for $20 \mathrm{~h}$. Analysis of crude ${ }^{1} \mathrm{H}$ NMR showed $>20: 1$ dimeta/other borylation products. Purification by evaporation of the solvent and addition of ether, followed by filtration and drying in vacuo, gave the title compound as a light brown solid (115 mg, $0.20 \mathrm{mmol}, 80 \%)$ : ${ }^{1} \mathrm{H}$ NMR (400 MHz, $\left.\mathrm{CDCl}_{3}\right) \delta 8.51(\mathrm{~s}, 1 \mathrm{H}), 8.06(\mathrm{~d}, J=12.9 \mathrm{~Hz}, 2 \mathrm{H}), 7.73(\mathrm{~d}, J=8.0 \mathrm{~Hz}$, $2 \mathrm{H}), 7.08(\mathrm{~d}, J=8.0 \mathrm{~Hz}, 2 \mathrm{H}), 2.41(\mathrm{~d}, J=14.4 \mathrm{~Hz}, 9 \mathrm{H}), 2.30(\mathrm{~s}, 3 \mathrm{H})$, $1.34(\mathrm{~s}, 24 \mathrm{H}) ;{ }^{13} \mathrm{C}\left\{{ }^{1} \mathrm{H}\right\} \mathrm{NMR}\left(101 \mathrm{MHz}, \mathrm{CDCl}_{3}\right) \delta 146.6\left(\mathrm{~d},{ }^{4} J_{\mathrm{C}-\mathrm{P}}=\right.$ $2.8 \mathrm{~Hz}), 143.8,138.9,138.3\left(\mathrm{~d},{ }^{2} J_{\mathrm{C}-\mathrm{P}}=9.8 \mathrm{~Hz}\right), 128.5,125.9,121.4$ $\left(\mathrm{d},{ }^{1} J_{\mathrm{C}-\mathrm{P}}=83.3 \mathrm{~Hz}\right), 84.7,24.9,21.3,9.8\left(\mathrm{~d},{ }^{1} J_{\mathrm{C}-\mathrm{P}}=56.1 \mathrm{~Hz}\right) ;{ }^{31} \mathrm{P}$ $\left(162 \mathrm{MHz}, \mathrm{CDCl}_{3}\right) \delta$ 22.34; HRMS (ESI+) calcd for $[\mathrm{M}-\mathrm{OTs}]^{+}$ $\left[\mathrm{C}_{21} \mathrm{H}_{36} \mathrm{~B}_{2} \mathrm{O}_{4} \mathrm{P}\right]^{+}$405.2532, found 405.2542.

4-Chloro-3-methylphenol (9). Following GP2, the compound was formed using (2-chlorobenzyl)trimethylphosphonium 4-methylbenzenesulfonate ( $3 \mathrm{~b})(372.8 \mathrm{mg}, 1.0 \mathrm{mmol}), \mathrm{B}_{2} \mathrm{Pin}_{2}(381 \mathrm{mg}, 1.5 \mathrm{mmol})$, $[\mathrm{Ir}(\mathrm{COD}) \mathrm{OMe}]_{2}(10 \mathrm{mg}, 0.015 \mathrm{mmol}), \mathbf{1}(15.2 \mathrm{mg}, 0.03 \mathrm{mmol})$, and 1,4-dioxane $(5 \mathrm{~mL})$. The reaction mixture was stirred for $16 \mathrm{~h}$ at 50 ${ }^{\circ} \mathrm{C}$. Analysis of crude ${ }^{1} \mathrm{H}$ NMR showed $>20: 1$ meta/para borylation. The solvent was removed; then the residue was dissolved in THF $(8.25 \mathrm{~mL})$ and methanol $(8.25 \mathrm{~mL}) . \mathrm{NaHCO}_{3}(1.05 \mathrm{~g}, 12.5$ equiv $)$ was added. and the mixture was cooled to $0{ }^{\circ} \mathrm{C}$ before dropwise addition of $\mathrm{H}_{2} \mathrm{O}_{2}(3 \mathrm{~mL}, 30 \%$ in water, $\sim 26 \mathrm{mmol})$. The resulting reaction mixture was stirred at room temperature for $1 \mathrm{~h}$ and then filtered through Celite, and the filtrate was concentrated under reduced pressure and dried under a high vacuum. The residue was dissolved in dry THF $(5 \mathrm{~mL})$ under an argon atmosphere, and the mixture was cooled to $0{ }^{\circ} \mathrm{C}$. A solution of lithium aluminum hydride $(2 \mathrm{~mL}, 1 \mathrm{M}$ in THF, $2 \mathrm{mmol}$ ) was added dropwise, and then the mixture was heated to $60^{\circ} \mathrm{C}$ for $1 \mathrm{~h}$. The reaction was cooled to $0{ }^{\circ} \mathrm{C}$ and quenched by the addition of water, then acidified using $1 \mathrm{M} \mathrm{HCl}$, and extracted with $\mathrm{CH}_{2} \mathrm{Cl}_{2}$. The organic layer was dried over $\mathrm{MgSO}_{4}$ and purified by column chromatography on silica gel (5\% EtOAc in petroleum ether $40-60)$ to afford 9 as a white solid $(65 \mathrm{mg}, 0.46$ mmol, $46 \%$ over 3 steps): ${ }^{1} \mathrm{H}$ NMR $\left(400 \mathrm{MHz}, \mathrm{CDCl}_{3}\right) \delta 7.18$ (d, $J=$ $8.5 \mathrm{~Hz}, 1 \mathrm{H}), 6.71(\mathrm{~d}, J=2.8 \mathrm{~Hz}, 1 \mathrm{H}), 6.61(\mathrm{dd}, J=8.6,3.0 \mathrm{~Hz}, 1 \mathrm{H})$, 5.14 (br, $1 \mathrm{H}), 2.31(\mathrm{~s}, 3 \mathrm{H}) ;{ }^{13} \mathrm{C}\left\{{ }^{1} \mathrm{H}\right\} \operatorname{NMR}\left(101 \mathrm{MHz}, \mathrm{CDCl}_{3}\right) \delta$ 153.9, 137.4, 129.8, 126.0, 117.8, 114.1, 20.1; HRMS (ESI+) calcd for $[\mathrm{M}-\mathrm{H}]^{-}\left[\mathrm{C}_{7} \mathrm{H}_{6} \mathrm{ClO}\right]^{-}$141.0113, found 141.0110. 


\section{ASSOCIATED CONTENT}

\section{S Supporting Information}

The Supporting Information is available free of charge on the ACS Publications website at DOI: 10.1021/acs.joc.9b00878.

${ }^{1} \mathrm{H}$ NMR and ${ }^{13} \mathrm{C}$ NMR spectra for all compounds (PDF)

\section{AUTHOR INFORMATION}

\section{Corresponding Author}

*E-mail: rjp71@cam.ac.uk.

ORCID $\odot$

Robert J. Phipps: 0000-0002-7383-5469

Notes

The authors declare no competing financial interest.

\section{ACKNOWLEDGMENTS}

We are grateful to AstraZeneca for a Ph.D. studentship through the AZ-Cambridge Ph.D. program (M.T.M.), the Royal Society for a University Research Fellowship (R.J.P.), the EPSRC (EP/N005422/1), and the ERC (StG 757381). We are very grateful to Dr. Holly J. Davis for performing initial experiments. We thank the EPSRC U.K. National Mass Spectrometry Facility at Swansea University as well as Professors Steven V. Ley and Matthew J. Gaunt for support and useful discussions.

\section{REFERENCES}

(1) (a) Dey, A.; Agasti, S.; Maiti, D. Palladium catalysed meta-C-H functionalization reactions. Org. Biomol. Chem. 2016, 14, 5440-5453. (b) Li, J.; De Sarkar, S.; Ackermann, L. meta- and para-Selective C-H Functionalization by $\mathrm{C}-\mathrm{H}$ Activation. In $\mathrm{C}-\mathrm{H}$ Bond Activation and Catalytic Functionalization I; Dixneuf, P. H., Doucet, H., Eds.; Springer International Publishing: Cham, 2016; pp 217-257. (c) Mihai, M. T.; Genov, G. R.; Phipps, R. J. Access to the meta position of arenes through transition metal catalysed $\mathrm{C}-\mathrm{H}$ bond functionalisation: a focus on metals other than palladium. Chem. Soc. Rev. 2018, 47, 149-171. (d) Dey, A.; Sinha, S. K.; Achar, T. K.; Maiti, D. Accessing Remote Meta- and Para-C-H Bonds With Covalently Attached Directing Groups. Angew. Chem., Int. Ed. 2019, 0, 1.

(2) (a) Davis, H. J.; Phipps, R. J. Harnessing non-covalent interactions to exert control over regioselectivity and site-selectivity in catalytic reactions. Chem. Sci. 2017, 8, 864-877. (b) Dydio, P.; Reek, J. N. H. Supramolecular control of selectivity in transition-metal catalysis through substrate preorganization. Chem. Sci. 2014, 5, 21352145. (c) Raynal, M.; Ballester, P.; Vidal-Ferran, A.; van Leeuwen, P. W. N. M. Supramolecular catalysis. Part 1: non-covalent interactions as a tool for building and modifying homogeneous catalysts. Chem. Soc. Rev. 2014, 43, 1660-1733.

(3) (a) Breslow, R.; Zhang, X.; Huang, Y. Selective Catalytic Hydroxylation of a Steroid by an Artificial Cytochrome P-450 Enzyme. J. Am. Chem. Soc. 1997, 119, 4535-4536. (b) Das, S.; Incarvito, C. D.; Crabtree, R. H.; Brudvig, G. W. Molecular Recognition in the Selective Oxygenation of Saturated C-H Bonds by a Dimanganese Catalyst. Science 2006, 312, 1941-1943.

(4) (a) Smejkal, T.; Breit, B. A Supramolecular Catalyst for Regioselective Hydroformylation of Unsaturated Carboxylic Acids. Angew. Chem., Int. Ed. 2008, 47, 311-315. (b) Dydio, P.; Detz, R. J.; Reek, J. N. H. Precise Supramolecular Control of Selectivity in the Rh-Catalyzed Hydroformylation of Terminal and Internal Alkenes. J. Am. Chem. Soc. 2013, 135, 10817-10828.

(5) For seminal reports on Ir-catalyzed borylation, see: (a) Cho, J.Y.; Tse, M. K.; Holmes, D.; Maleczka, R. E.; Smith, M. R. Remarkably Selective Iridium Catalysts for the Elaboration of Aromatic C-H Bonds. Science 2002, 295, 305-308. (b) Ishiyama, T.; Takagi, J.; Ishida, K.; Miyaura, N.; Anastasi, N. R.; Hartwig, J. F. Mild Iridium-
Catalyzed Borylation of Arenes. High Turnover Numbers, Room Temperature Reactions, and Isolation of a Potential Intermediate. J. Am. Chem. Soc. 2002, 124, 390-391. (c) Takagi, J.; Sato, K.; Hartwig, J. F.; Ishiyama, T.; Miyaura, N. Iridium-catalyzed $\mathrm{C}-\mathrm{H}$ coupling reaction of heteroaromatic compounds with bis(pinacolato)diboron: regioselective synthesis of heteroarylboronates. Tetrahedron Lett. 2002, 43, 5649-5651. (d) Boller, T. M.; Murphy, J. M.; Hapke, M.; Ishiyama, T.; Miyaura, N.; Hartwig, J. F. Mechanism of the Mild Functionalization of Arenes by Diboron Reagents Catalyzed by Iridium Complexes. Intermediacy and Chemistry of BipyridineLigated Iridium Trisboryl Complexes. J. Am. Chem. Soc. 2005, 127, 14263-14278. For leading reviews on Ir-catalyzed borylation, see: (e) Mkhalid, I. A. I.; Barnard, J. H.; Marder, T. B.; Murphy, J. M.; Hartwig, J. F. C-H Activation for the Construction of $\mathrm{C}-\mathrm{B}$ Bonds. Chem. Rev. 2010, 110, 890-931. (f) Hartwig, J. F. Regioselectivity of the borylation of alkanes and arenes. Chem. Soc. Rev. 2011, 40, 19922002. (g) Ros, A.; Fernandez, R.; Lassaletta, J. M. Functional group directed C-H borylation. Chem. Soc. Rev. 2014, 43, 3229-3243.

(6) (a) Roosen, P. C.; Kallepalli, V. A.; Chattopadhyay, B.; Singleton, D. A.; Maleczka, R. E.; Smith, M. R. Outer-Sphere Direction in Iridium C-H Borylation. J. Am. Chem. Soc. 2012, 134, 11350-11353. (b) Preshlock, S. M.; Plattner, D. L.; Maligres, P. E.; Krska, S. W.; Maleczka, R. E.; Smith, M. R. A Traceless Directing Group for C-H Borylation. Angew. Chem., Int. Ed. 2013, 52, 1291512919. (c) Kuninobu, Y.; Ida, H.; Nishi, M.; Kanai, M. A metaselective $\mathrm{C}-\mathrm{H}$ borylation directed by a secondary interaction between ligand and substrate. Nat. Chem. 2015, 7, 712-717. (d) Chattopadhyay, B.; Dannatt, J. E.; Andujar-De Sanctis, I. L.; Gore, K. A.; Maleczka, R. E.; Singleton, D. A.; Smith, M. R. Ir-Catalyzed orthoBorylation of Phenols Directed by Substrate-Ligand Electrostatic Interactions: A Combined Experimental/in Silico Strategy for Optimizing Weak Interactions. J. Am. Chem. Soc. 2017, 139, 78647871. (e) Hoque, M. E.; Bisht, R.; Haldar, C.; Chattopadhyay, B. Noncovalent Interactions in Ir-Catalyzed $\mathrm{C}-\mathrm{H}$ Activation: L-Shaped Ligand for Para-Selective Borylation of Aromatic Esters. J. Am. Chem. Soc. 2017, 139, 7745-7748. (f) Davis, H. J.; Genov, G. R.; Phipps, R. J. meta-Selective $\mathrm{C}-\mathrm{H}$ Borylation of Benzylamine-, Phenethylamine-, and Phenylpropylamine-Derived Amides Enabled by a Single Anionic Ligand. Angew. Chem., Int. Ed. 2017, 56, 13351-13355. (g) Bisht, R.; Hoque, M. E.; Chattopadhyay, B. Amide Effects in C-H Activation: Noncovalent Interactions with L-Shaped Ligand for meta Borylation of Aromatic Amides. Angew. Chem., Int. Ed. 2018, 57, 15762-15766. (h) Wang, J.; Torigoe, T.; Kuninobu, Y. Hydrogen-Bond-Controlled Formal Meta-Selective $\mathrm{C}-\mathrm{H}$ Transformations and Regioselective Synthesis of Multisubstituted Aromatic Compounds. Org. Lett. 2019, 21, 1342-1346. (i) Lu, X.; Yoshigoe, Y.; Ida, H.; Nishi, M.; Kanai, M.; Kuninobu, Y. Hydrogen Bond-Accelerated meta-Selective $\mathrm{C}-\mathrm{H}$ Borylation of Aromatic Compounds and Expression of Functional Group and Substrate Specificities. ACS Catal. 2019, 9, 1705-1709.

(7) For examples of phosphine-directed borylation, see: (a) Crawford, K. M.; Ramseyer, T. R.; Daley, C. J. A.; Clark, T. B. PhosphineDirected C-H Borylation Reactions: Facile and Selective Access to Ambiphilic Phosphine Boronate Esters. Angew. Chem., Int. Ed. 2014, 53, 7589-7593. (b) Fukuda, K.; Iwasawa, N.; Takaya, J. RutheniumCatalyzed ortho $\mathrm{C}-\mathrm{H}$ Borylation of Arylphosphines. Angew. Chem. 2019, 131, 2876-2879. (c) Wen, J.; Wang, D.; Qian, J.; Wang, D.; Zhu, C.; Zhao, Y.; Shi, Z. Rhodium-Catalyzed PIII-Directed ortho$\mathrm{C}-\mathrm{H}$ Borylation of Arylphosphines. Angew. Chem., Int. Ed. 2019, 58, 2078-2082.

(8) (a) Breslow, R.; Rajagopalan, R.; Schwarz, J. Selective functionalization of doubly coordinated flexible chains. J. Am. Chem. Soc. 1981, 103, 2905-2907. (b) Breslow, R.; Heyer, D. Directed steroid chlorination catalyzed by an ion-paired template. Tetrahedron Lett. 1983, 24, 5039-5042.

(9) (a) Davis, H. J.; Mihai, M. T.; Phipps, R. J. Ion Pair-Directed Regiocontrol in Transition-Metal Catalysis: A Meta-Selective $\mathrm{C}-\mathrm{H}$ Borylation of Aromatic Quaternary Ammonium Salts. J. Am. Chem. Soc. 2016, 138, 12759-12762. (b) Mihai, M. T.; Davis, H. J.; Genov, G. R.; Phipps, R. J. Ion Pair-Directed C-H Activation on Flexible 
Ammonium Salts: meta-Selective Borylation of Quaternized Phenethylamines and Phenylpropylamines. ACS Catal. 2018, 8, 3764-3769.

(10) Byrne, P. A.; Gilheany, D. G. The modern interpretation of the Wittig reaction mechanism. Chem. Soc. Rev. 2013, 42, 6670-6696.

(11) (a) McNulty, J.; Das, P. Highly Stereoselective and General Synthesis of (E)-Stilbenes and Alkenes by Means of an Aqueous Wittig Reaction. Eur. J. Org. Chem. 2009, 2009, 4031-4035.

(b) McNulty, J.; Das, P. Aqueous Wittig reactions of semi-stabilized ylides. A straightforward synthesis of 1,3-dienes and 1,3,5-trienes. Tetrahedron Lett. 2009, 50, 5737-5740. (c) Das, P.; McLeod, D.; McNulty, J. A direct synthesis of functionalized styrenes and terminal 1,3-dienes via aqueous Wittig chemistry with formalin. Tetrahedron Lett. 2011, 52, 199-201.

(12) (a) Hwang, L. K.; Na, Y.; Lee, J.; Do, Y.; Chang, S. Tetraarylphosphonium Halides as Arylating Reagents in Pd-Catalyzed Heck and Cross-Coupling Reactions. Angew. Chem., Int. Ed. 2005, 44, 6166-6169. (b) Zhang, X.; McNally, A. Phosphonium Salts as Pseudohalides: Regioselective Nickel-Catalyzed Cross-Coupling of Complex Pyridines and Diazines. Angew. Chem., Int. Ed. 2017, 56, 9833-9836.

(13) Bailey, W. J.; Buckler, S. A. Phosphorus Compounds. I. Reduction of Benzylphosphonium Compounds with Lithium Aluminum Hydride. J. Am. Chem. Soc. 1957, 79, 3567-3569. 